\title{
Sulla rappresentazione tipica delle forme binarie.
}

\author{
(dei Professori A.Clebsch e P. Gordna, a Giessen).
}

$U_{N A}$ forma binaria di grado $2 n+1 \quad(n>1)$ può essere rappresentata tipicamente, ossia con coefficienti che siano a un tempo invarianti, se, deviando alquanto dal metodo adottato dal sig. ${ }^{\mathrm{r}}$ Hermite per le forme di quinto grado, si introducono due covarianti lineari come variabili. Siccome però il numero dei coefficienti della forma tipica, incluso un denominatore comune, supera di quattro il numero degli invarianti, così si possono introdurre i coefficienti medesimi della forma tipica come invarianti fondamentali, purchè si stabiliscano fra essi quattro relazioni, le quali diano a quella il carattere di forma tipica. Ciò si effettua costruendo per la forma tipica i covarianti scelti come variabili e scrivendo le condizioni perchè essi coincidano colle variabili stesse, moltiplicate per una potenza (facile a riconoscersi) del determinante della trasformazione. Questo processo perd non è più sufficiente quando si voglia esprimere tutto mediante gli invarianti più semplici della forma. In tal caso conviene seguire vie speciali; e la forma tipica può essere ottenuta con mezzi che, se anche affatto simili fra loro, pure presentano qualche differenza pei diversi gradi. Noi effettueremo questa rappresentazione per le forme di quinto grado; la quale a un di presso comprende in sè tutta la teoria di queste forme; e si rileverà facilmente che le forme di grado dispari possono, l'una dopo l'altra, cssere trattate nella stessa guisa.

Similmente per le forme di grado $2 n(n>2)$. Qui si ottiene la rappresentazione tipica introducendo tre covarianti di secondo grado; fra questi ha luogo una equazione di secondo grado, mentre la forma stessa è rappresentata come forma ternaria del grado $n$, ed i coefficienti sono tutti 
invarianti. Anche in questo caso si possono assumere i coefficenti come invarianti fondamentali, cercando le relazioni fra i medesimi le quali caratterizzano la forma tipica. Per ottenere queste relazioni basta comporre per la forma tipica i prescelti covarianti di secondo grado e paragonarli con quelli assunti come dati. Ed anche qui occorrono metodi particolari per esprimere il tutto mediante gli invarianti più semplici. Noi li svilupperemo, in via d'esempio, per le forme di sesto grado, e daremo i tratti principali della teoria di queste forme; il proseguire la via analoga per le forme di grado superiore non ha alcuna difficoltà.

I risultati ottenuti, in quanto risguardano le forme di quinto grado, 0 sono già noti pei lavori dei signori Cayley, Sylvester, Salmon, Hermite, o facilmente deducibili da essi; noi crediamo quindi che per questa parte l' importanza principale stia nell' esposizione sistematica. Ma rispetto alle forme di sesto grado sono state assegnate finora soltanto alcune forme fondamentali (CAYley, Memoirs upon Quantics) ed in ispecie calcolati gli invarianti.(cfr. Salmon, Higher Algebra, 2. ${ }^{a}$ ediz.), senza che siasi tentato di presentare la connessione delle forme, il che invece noi facciamo completamente. Del resto gli invarianti qui prescelti come fondamentali differiscono da quelli usati altrove, essendo nella presente ricerca emersa da sè la necessità di introdurre appunto questi e non altri invarianti.

$\$ 1$.

Forme di quinto grado. Loro forme fondamentall.

La forma binaria:

$$
u=a x_{1}^{5}+5 b x_{1}^{4} x_{2}+10 c x_{2}^{8} x_{2}^{2}+10 d x_{1}^{2} x_{2}^{3}+5 e x_{1} x_{2}^{4}+f x_{2}^{3}
$$

si rappresenti simbolicamente con :

$$
u=a_{x}^{\prime}=a_{x}^{\prime \prime}=\cdots\left(a_{x}=a_{1} x_{1}+a_{2} x_{2}, a_{x}^{\prime}=a_{1}^{\prime} x_{1}+a_{2}^{\prime} x_{2}, \ldots\right)
$$

Ricavando da $u$ la forma di quarto grado nelle $y$ :

$$
\frac{1}{s}\left(x_{1} \frac{\partial u(y)}{\partial y_{1}}+x_{2} \frac{\partial u(y)}{\partial y_{2}}\right)=a_{y}^{i} \cdot a_{x}
$$


e formando rispetto ad essa gli invarianti $i, j$ della forma di quarto grado, si ottengono covarianti di secondo e terzo grado. Se con $u_{i}, u_{i k}$ si indicano sempre derivate divise per $\nu, v(v-1), \ldots$, dove $\nu$ è il grado della funzione, sarà :

$$
\begin{aligned}
& i=2\left\{u_{1111} u_{2222}-4 u_{1112} u_{1222}+3 u_{1122}^{2}\right\}, \\
& j=3\left|\begin{array}{lll}
u_{111} & u_{1112} & u_{1122} \\
u_{1112} & u_{1122} & u_{1222} \\
u_{1122} & u_{1222} & u_{2222}
\end{array}\right|,
\end{aligned}
$$

orvero simbolicamente, posto $a_{i} b_{k}-b_{i} a_{k}=(a, b)$ :

$$
\begin{aligned}
& i=i_{x}^{2}=i_{x}^{\prime 2} \cdots=\left(a a^{\prime}\right)^{4} a_{x} a_{x}^{\prime}, \\
& j=j_{x}^{2}=j_{x}^{\prime 2} \cdots=\frac{1}{2}\left(a a^{\prime}\right)^{2}\left(a^{\prime} a^{\prime \prime}\right)^{2}\left(a^{\prime \prime} a\right)^{2} a_{x} a_{x}^{\prime} a^{\prime \prime}{ }_{x} .
\end{aligned}
$$

L'espressione simbolica di $j$ può essere trasformata, e si arriva così ad un'altra maniera di generare $j$. In fatti, se si fa uso dell'equazione simbolica:

$$
\left(a a^{\prime}\right) a_{x}^{\prime \prime}=\left(a a^{\prime \prime}\right) a_{x}^{\prime}-\left(a^{\prime} a^{\prime \prime}\right) a_{x},
$$

$j$ si muta nella somma di due termini che collo scamkio di $a^{\prime}$ ed a diventano perfettamente egualj, onde si puo invece dei due termini medesimi porre il doppio dell'uno di essi. Si ha cosi:

$$
j=-\left(a a^{\prime}\right)\left(a^{\prime} a^{\prime \prime}\right)^{3}\left(a a^{\prime \prime}\right)^{2} a_{x}^{2} a_{x}^{\prime} .
$$

Sostituendo di nuovo per $\left(a a^{\prime \prime}\right) a_{x}^{\prime}$ il suo valore desunto dall'identita superiore, $j$ si divide ancora in due parti:

$$
j=-\left(a a^{\prime}\right)\left(a a^{\prime \prime}\right)\left(a^{\prime} a^{\prime \prime}\right)^{4} a_{x}^{3}-\left(a a^{\prime}\right)^{2}\left(a a^{\prime \prime}\right)\left(a^{\prime} a^{\prime \prime}\right)^{3} a_{x}^{2} a_{x}^{\prime \prime}
$$

la seconàa delle quali è $-j$, perchè essa si desume dall'espressione precedente scambiando $a^{\prime}$ con $a^{\prime \prime}$; e la prima è, con altro segno, l'espressione simbolica della funzione $u_{11} i_{22}-2 u_{12} i_{12}+u_{22} i_{11} ;$ si ha dunque la formola:

$$
u_{11} i_{22}-2 u_{12} i_{12}+u_{22} i_{11}=-2 j
$$

che somministra una seconda maniera di formare $j$. 
Ma fra $u$ e $j$ sussiste ancora un'altra relazione che è di importanza fondamentale per la teoria. Ciò si annulla identicamente il covariante di secondo grado :

$$
u_{111} j_{222}-3 u_{112} j_{122}+3 u_{122} j_{112}-u_{222} j_{111}=\left(a_{i j}\right)^{3} a_{x}^{2}
$$

In fatti, se si pone per $j$ l'espressione simbolica:

$$
j=-\frac{1}{2}(a i)^{2} a_{x}^{3} .
$$

che risulta dalla sua seconda rappresentazione, il covariante anzidetto assume la forma:

$$
(a i)^{2} \cdot\left(a a^{\prime}\right)^{3} \cdot a_{x}^{\prime 2}=\frac{1}{2}\left(a \alpha^{\prime}\right)^{3}\left\{(a i)^{2} a_{x}{ }^{2}-\left(a^{\prime} i\right)^{2} a_{x}^{2}\right\},
$$

la quale, per l'identità già considerata:

$$
(a i) a_{x}^{\prime}-\left(a^{\prime} i\right) a_{x}=\left(a, a^{\prime}\right) i_{x}
$$

diviene:

$$
\frac{1}{2}\left(a a^{\prime}\right)^{4} i_{x}\left\{(a i) a_{x}^{\prime}+\left(a^{\prime} i\right) a_{x}\right\} .
$$

Ma questa non è altra cosa che il determinante funzionale di due funzioni entrambe uguali ad $i$, epperò è identicamente zero.

Da $j$ scaturisce un secondo covariante quadratico:

$$
\tau=2\left(j_{11} j_{22}-j_{12}^{2}\right)=2\left|\begin{array}{ccc}
j_{111} & j_{112} & x_{2}^{2} \\
j_{112} & j_{122} & -x_{1} x_{2} \\
j_{122} & j_{222} & x_{1}^{2}
\end{array}\right|=\tau_{x c}^{2} \cdots=\left(j j^{\prime}\right)^{2} j_{x_{1}} j_{x}{ }^{\prime}
$$

Se questo covariante si combina con $i$ nella forma $\tau+\lambda i$, nei coefficienti del determinante di questa funzione si otterranno gli invarianti indipendenti di $u$. Cioè, se si pone:

$$
\left|\begin{array}{cc}
\tau_{11}+\lambda i_{11} & \tau_{12}+\lambda i_{12} \\
\tau_{12}+\lambda i_{12} & \tau_{22}+\lambda i_{22}
\end{array}\right|=\lambda^{2} A+2 \lambda B+C,
$$

i tre invarianti (i cui ordini sono rispettivamente 4,8 e 12) sarauno: 


$$
\begin{aligned}
& A=i_{11} i_{22}-i_{12}^{2}=\frac{1}{2}\left(i i^{\prime}\right)^{2}, \\
& B=\frac{1}{2}\left(i_{11} \tau_{22}-2 i_{12} \tau_{12}+i_{22} \tau_{11}\right)=-\frac{1}{2}(i \tau)^{2} . \\
& G=\tau_{11} \tau_{22}-\tau_{12}^{2}=\frac{1}{2}\left(\tau \tau^{\prime}\right)^{2} .
\end{aligned}
$$

Il divisore 2 in $B$ non è che apparente, perchè, in virtù del modo con cui sono formati, $i_{11}, i_{22}, \tau_{11}, \tau_{22}$ sono divisibili per 2 .

Inoltre da $i$ e da $\tau$ si ricava il covariante:

$$
\lesssim=i_{1} \tau_{2}-\tau_{1} i_{2}=(i \tau) i_{x} \tau_{x}=\left|\begin{array}{ccc}
i_{11} & \tau_{11} & x_{2}^{\prime 2} \\
i_{12} & \tau_{12} & -x_{1} x_{2} \\
i_{22} & \tau_{22} & x_{1}^{2}
\end{array}\right| .
$$

L'ultima espressione di \& mostra a dirittura che hanno luogo le due equazioni:

$$
\begin{aligned}
& \S_{11} i_{22}-2 \S_{12} i_{12}+\S_{22} i_{11}=0, \\
& \bigotimes_{11} \tau_{22}-2 \S_{12} \tau_{12}+\$_{22} \tau_{11}=0 .
\end{aligned}
$$

Ia il determinante di $\$$ :

$$
\frac{1}{2}\left(\vartheta_{11} \curvearrowright_{22}-2 \$_{12} \curvearrowright_{12}+\$_{22} \&_{11}\right)
$$

e può per conseguenza essere riprodottn dalla moltiplicazione dei determinanti corrispondenti dei sistemi incompleti:

$$
\left|\begin{array}{ccc}
i_{11} & i_{12} & i_{22} \\
\tau_{11} & \tau_{12} & \tau_{22}
\end{array}\right|\left|\begin{array}{lll}
i_{22} & -2 i_{12} & i_{11} \\
\tau_{22} & -2 \tau_{12} & \tau_{11}
\end{array}\right|
$$

che dà il determinante:

$$
\frac{1}{4}\left|\begin{array}{ll}
i_{11} i_{22}-2 i_{12} i_{12}+i_{22} i_{11} & i_{11} \tau_{22}-2 i_{12} \tau_{12}+i_{22} \tau_{11} \\
\tau_{11} i_{22}-2 \tau_{12} i_{12}+\tau_{22} i_{11} & \tau_{11} \tau_{22}-2 \tau_{12} \tau_{12}+\tau_{22} \tau_{11}
\end{array}\right| .
$$

Si ha dunque la formola:

$$
\$_{11} \$_{22}-\$_{12}^{2}=A C i-B^{2}
$$


Per rappresentare $S^{2}$ in $i$ e $\tau$, si ha in virtù dell' espressione di $\$$ già adoperata:

$$
\$^{2}=\frac{1}{2}\left|\begin{array}{ccc}
i_{11} & i_{12} & i_{22} \\
\tau_{11} & \tau_{12} & \tau_{22} \\
x_{2}^{2} & -x_{1} x_{2} & x_{1}^{2}
\end{array}\right|\left|\begin{array}{ccc}
i_{29} & -2 i_{12} & i_{11} \\
\tau_{22} & -2 \tau_{12} & \tau_{11} \\
x_{1}^{2} & 2 x_{1} x_{2} & x_{2}^{2}
\end{array}\right|
$$

ossia, eseguendo la moltiplicazione :

$$
\Im^{2}=\left|\begin{array}{ccc}
A & B & i \\
B & C & \tau \\
i & \tau & 0
\end{array}\right|
$$

e finalmente :

$$
\$^{2}=-\left\{A \tau^{2}-2 B \tau i+G i^{2}\right\}
$$

Differenziando questa equazione si trova:

$$
\begin{aligned}
& \$ \$_{1}=i_{1}(B \tau-C i)-\tau_{1}(A \tau-B i), \\
& \$ \$_{2}=i_{2}(B \tau-C i)-\tau_{2}(A \tau-B i),
\end{aligned}
$$

e quindi :

$$
\begin{aligned}
& \$_{1} i_{2}-\$_{2} i_{1}=A \tau-B i, \\
& \vartheta_{1} \tau_{2}-\$_{2} \tau_{1}=B \tau-C i .
\end{aligned}
$$

Da ultimo ritornando alle forme $i$ e $j$, dalla combinazione delle medesime nasce an covariante lineare:

$$
\alpha=a_{1} x_{1}+\alpha_{2} x_{2}=\frac{1}{2}\left(i_{11} j_{22}-2 i_{12} j_{12}+i_{22} j_{22}\right)=\frac{1}{2}(i j)^{2} j_{x}=-\frac{1}{4}(a i)^{2}\left(a i^{\prime}\right)^{2} a_{x} .
$$

Altri covarianti lineari si formano combinando $\alpha$ con $i, \tau, \$()$; di alcuni fra essi si trattera ancora più avanti.

(') Formando il determinante funzionale di ciascun covariante quadratico $i$ con ciaseun covariante $\varphi$ di grado $v$, si genera un altro covariante dello stesso grado. Ma si possono formare nuovi covarianti dello stesso grado $\vee$ anche differenziando $q$ rispetto ai coeflicienti della funzione primitiva $u$ di grado $n$ e mettendo invece delle differen- 
$\S 2$.

\section{Relazioni fra gli invarianti.}

L'equazione:

$$
\aleph^{2}=-\left\{A \tau^{2}-2 B \tau i+C i^{2}\right\}
$$

somministra incontanente la rappresentazione del quadrato dell' invariante (di Hermite) del $18 .^{\circ}$ ordine mediante gli invarianti $A, B, C$. In fatti, se in $\$, \tau$ ed $i$ poniamo per $x_{1}, x_{2}$ i coefficienti $\alpha_{2},-\alpha_{1}$ del covariante lineare $\alpha$, allora $\&$ si cambia nell' invariante del $18 .^{\circ}$ ordine:

$$
2 R=\left|\begin{array}{lll}
i_{11} & \tau_{11} & \alpha_{1}^{2} \\
i_{12} & \tau_{12} & \alpha_{1} \alpha_{2} \\
i_{22} & \tau_{22} & \alpha_{2}^{2}
\end{array}\right|=\bigotimes_{11} \alpha_{1}^{2}-2 \S_{12} \alpha_{1} \alpha_{2}+\S_{22} \alpha_{2}^{2},
$$

ziali i coefficienti della forma $u_{1} i_{2} \rightarrow i_{1} u_{2}$, che è parimente di grado $n$. Allora si prosenta la notabile circostanza che $\mathrm{i}$ due processi conduçono al medesimo risultato. Sia in fatti la funzione data:

$$
u=a_{0} x_{1}^{n 2}+n \cdot a_{1} x_{1}^{n-1} x_{2}+\frac{n \cdot n-1}{2} a_{2} x_{1}^{n-2} x_{2}^{2}+\ldots ;
$$

le equazioni differenziali parziali soddisfatte dal covariante $\varphi$ si possono, secondo il signor AronhoLd, porre sotto la forma simbolica:

$$
\begin{array}{lll}
\xi_{1} \frac{\partial u(\xi)}{\partial \xi_{1}}-x_{1} \frac{\partial \varphi}{\partial x_{1}}=\lambda \varphi, & \xi_{2} \frac{\partial u(\xi)}{\partial \xi_{1}}-x_{2} \frac{\partial \varphi}{\partial x_{1}}=0, \\
\xi_{1} \frac{\partial u(\xi)}{\partial \xi_{2}}-x_{1} \frac{\partial \varphi}{\partial x_{2}}=0, & \xi_{2} \frac{\partial u(\xi)}{\partial \xi_{2}}-x_{2} \frac{\partial \varphi}{\partial x_{2}}=\lambda \varphi,
\end{array}
$$

introducendo le equazioni simboliche:

$$
\frac{\partial \varphi}{\partial a_{0}}=\dot{\xi}_{1}^{n}, \quad \frac{\partial \varphi}{\partial a_{1}}=n \cdot \xi_{1}^{n-1} \xi_{2}, \quad \frac{\partial \varphi}{\partial a_{2}}=\frac{n \cdot n-1}{2} \xi_{1}^{n-2} \xi_{2}^{2}, \text { ecc. }
$$

Indicati ora con $\alpha_{0}, \alpha_{1}, \ldots$ i coefficienti della forma $u_{1} i_{2}-i_{2} u_{1}$, corrispondenti agli $a$, consideriamo la nuova forma nascente da $\varphi$ :

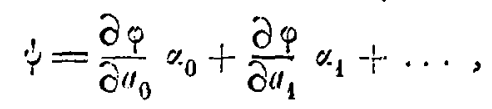


mentre $i$ e $\tau$ diventano gli invarianti del $12 .^{\circ}$ e del $160^{\circ}$ ordine:

$$
\begin{aligned}
& 2 M=i_{11} \alpha_{2}^{2}-2 i_{12} \alpha_{1} \alpha_{2}+i_{22} \alpha_{1}^{2}, \\
& 2 N=\tau_{11} \alpha_{2}^{2}-2 \tau_{12} \alpha_{1} \alpha_{2}+\tau_{22} \alpha_{1}^{2} .
\end{aligned}
$$

Dunque dall'equazione superiore si ha la relazione fra $R, M, N$ :

$$
l^{2}=-\left\{A N^{2}-2 B M N+C M^{2}\right\}
$$

Resta soltanto ad esprimere $M$ ed $N$ per mezzo di $A, B, G$, ció che si fa razionalmente.

Se le forme $a, i, j$, che sono rispettivamente del primo, secondo e terzo grado, si riguardano come indipendenti fra loro, competono ad esse cinque invarianti indipendenti i quali sono gli invarianti $A, B, C, M, N$.

Se si fa uso solamente della circostanza che $\alpha$ nasce da $i$ e $j$, considerando però ancora $i$ coefficienti di $i$ e $j$ come indipendenti, ne segue una relazione fra $i$ cinque invarianti, ed in vero si vede facilmente che essa consiste in una rappresentazione di $N$ per mezzo di $A, B, C$. Si ottiene questa relazione nel modo seguente.

La forma $N$ non è che $\frac{1}{2} \tau$, dove $x_{1}, x_{2}$ siano surrogate da $\alpha_{2},-\alpha_{1}$, cioè si ha:

$$
N=\left|\begin{array}{ll}
j_{111} \alpha_{2}-j_{112} \alpha_{1} & j_{112} \alpha_{2}-j_{122} \alpha_{1} \\
j_{112} \alpha_{2}-j_{122} \alpha_{1} & j_{122} \alpha_{2}-j_{222} \alpha_{1}
\end{array}\right|,
$$

la quale per mezzo delle sostituzioni simboliche si trasforma manifestamente in:

$$
\alpha_{0} \varkappa_{1}^{n}+n \cdot \psi_{1} \xi_{1}^{n-1} \xi_{2}+\ldots=\left(u_{1} i_{2}-i_{2} u_{1}\right)_{\hat{\xi}}
$$

per modo che si ha simbolicamente:

$$
\dot{y}=\left(i_{12} \zeta_{1}+i_{22} \ddot{\xi}_{2}\right) u_{1}(\dot{\zeta})-\left(i_{11} \ddot{\zeta}_{1}+i_{12} \ddot{\zeta}_{2}\right) u_{2}(\ddot{\xi})
$$

ovvero, per le equazioni differenziali parziali soddisfatte da $\varphi$ :

Si ha dunque il teorema:

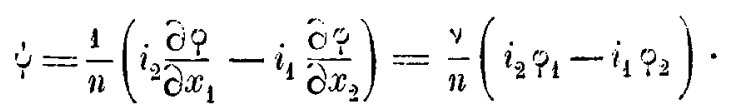

Se si differenzia un covariante $\varphi$ di grado $v$ rispetto ai coefficienti della funzione $u$ di grado $n$, dalla quale esso trae origine, e se invece delle differenziali si pongono $\mathrm{i}$ coefficienti della forma $u_{1} i_{2}-i_{1} u_{2}$, dove $i$ è un covariante quadratico qualsivoglia, si ottiene il covariante $\phi_{1} i_{2}-i_{1} i_{2}$ moltiplicato per $\frac{\nu}{n}$. 
vale a dire, $N$ è il determinante della forma di secondo grado:

$$
j_{1} \alpha_{2}-j_{2} \alpha_{1}=j_{x}^{2}(j \alpha),
$$

la quale, mercè l'introduzione dell' espressione simbolica di $a$ assume successivamente le forme:

$$
\begin{aligned}
j_{x}^{2}(j \alpha) & =\frac{1}{2} j_{x}^{9}\left(j j^{\prime}\right)\left(j^{\prime} i\right)^{2} \\
& =\frac{1}{1}\left(j j^{\prime}\right)\left\{j_{x}^{2}\left(j^{\prime} i\right)^{2}-j_{x}^{\prime 2}(j i)^{2}\right\} \\
& =-\frac{1}{4}\left(j j^{\prime}\right)^{2} i_{x}\left\{(j i) j_{x}^{\prime}+\left(j^{\prime} i\right) j_{x}\right\} \\
& =\frac{1}{2}(i \tau) i_{x} \widetilde{\tau}_{x}=\frac{1}{2} \Omega .
\end{aligned}
$$

Dunque $N$ è il determinante di \&, diviso per 4 , cioè si ha:

$$
N=\frac{1}{4}\left(A C-B^{2}\right) \cdot
$$

Se poi, in secondo luogo, si considera inoltre che $i$ e $j$ sono composti coi coefficienti della medesima forma di quinto grado, si ottiene una seconda relazione che serve ad esprimere $M$ per mezzo di $A, B, C$.

A quest'uopo cominciamo dal considerare la forma cubica:

$$
v=u_{11} \tau_{22}-2 u_{12} \tau_{12}+u_{22} \tau_{11}=a_{x}^{3}(a \tau)^{2} .
$$

che è di un'importanza primaria anche per ciò che segue. Il prodotto:

$$
A j=\frac{1}{4}\left(i i^{\prime}\right)^{2}\left(a a^{\prime}\right)^{2}\left(a a^{\prime \prime}\right)^{2}\left(a^{\prime} a^{\prime \prime}\right)^{2} a_{x} a_{x}^{\prime} a_{x}^{\prime \prime},
$$

per mezzo dell'equazione :

$$
\left(i i^{\prime}\right)^{2}\left(a a^{\prime}\right)^{2}=\left\{(a i)\left(a^{\prime} i^{\prime}\right)-\left(a^{\prime} i\right)\left(a i^{\prime}\right)\right\}^{2},
$$

che scaturisce dalla relazione identica già usata più volte, e riunendo i tormini identici, si trasforma in :

$$
A j=\frac{1}{2}\left\{(a i)^{2}\left(a^{\prime} i^{\prime}\right)^{2}-(\alpha i)\left(a i^{\prime}\right)\left(a^{\prime} i\right)\left(a^{\prime} i^{\prime}\right)\right\}\left(\alpha a^{\prime \prime}\right)^{2}\left(a^{\prime} a^{\prime \prime}\right)^{2} a_{x} a_{x}^{\prime} \alpha_{x}^{\prime \prime} .
$$

Inoltre dalle identità :

$$
\begin{aligned}
& (a i)\left(a^{\prime} a^{\prime \prime}\right)+\left(a a^{\prime}\right)\left(a^{\prime \prime} i\right)+\left(a a^{\prime \prime}\right)\left(i a^{\prime}\right)=0, \\
& \left(a i^{\prime}\right)\left(a^{\prime} a^{\prime \prime}\right)+\left(a a^{\prime}\right)\left(a^{\prime \prime} i^{\prime}\right)+\left(a a^{\prime \prime}\right)\left(i^{\prime} a^{\prime}\right)=0
\end{aligned}
$$


seguono le equazioni:

$$
\begin{aligned}
& 2(a i)\left(a^{\prime} i\right)\left(a a^{\prime \prime}\right)\left(a^{\prime} a^{\prime \prime}\right)=(a i)^{2}\left(a^{\prime} a^{\prime \prime}\right)^{2}+\left(a^{\prime} i\right)^{2}\left(a a^{\prime \prime}\right)^{2}-\left(a a^{\prime}\right)^{2}\left(a^{\prime \prime} i\right)^{2}, \\
& 2\left(a i^{\prime}\right)\left(a^{\prime} i^{\prime}\right)\left(a a^{\prime \prime}\right)\left(a^{\prime} a^{\prime \prime}\right)=\left(a i^{\prime}\right)^{2}\left(a^{\prime} a^{\prime \prime}\right)^{2}+\left(a^{\prime} i^{\prime}\right)^{2}\left(a a^{\prime \prime}\right)^{2}-\left(a a^{\prime}\right)^{2}\left(a^{\prime \prime} i^{\prime}\right)^{2}
\end{aligned}
$$

e trasformando, mediante queste equazioni, la seconda parte dell'espressione precedente di $A j$, si trova, dopo aver riuniti i termini identici:

$$
A j=-\frac{3}{8}(a i)^{2}\left(a i^{\prime}\right)^{2}\left(a^{\prime} a^{\prime \prime}\right)^{4} a_{x} a_{x}^{\prime} a_{x}^{\prime \prime}+\frac{3}{4}(a i)^{2}\left(a^{\prime} i^{\prime}\right)^{2}\left(a a^{\prime \prime}\right)^{2}\left(a^{\prime} a^{\prime \prime}\right)^{2} a_{x} a_{x}^{\prime} a_{x}^{\prime \prime} .
$$

La prima parte del seconda membro non è altro che $\frac{3}{2} \alpha i$; la seconda parte, in virtù della seconda formola per $j$, può essere surrogata da:

$$
3(j a)^{2}\left(j^{\prime} a\right)^{2} a_{x} j_{x} j_{x}^{\prime},
$$

e quindi trasformata mediante l'equazione:

$$
\text { (ja) } j_{x}^{\prime}=\left(j j^{\prime}\right) a_{x}-\left(j^{\prime} a\right) j_{j}
$$

omettendovi la seconda parte contenente $\left(j^{\prime}(a)^{3}\right.$, a cagione della relazione fra $u$ e $j$. Si trova così per quella parte l'espressione:

$$
\begin{gathered}
3(j a)\left(j^{\prime} a\right)^{2}\left(j j^{\prime}\right) a_{x}^{2} j_{x}=\frac{3}{2}(j a)\left(j^{\prime} a\right)\left(j j^{\prime}\right) a_{x}^{2}\left\{\left(j^{\prime} a\right) j_{x}-(j a) j_{x}^{\prime}\right\} \\
=-\frac{3}{2}(j a)\left(j^{\prime}(a)\left(j j^{\prime}\right)^{2} a_{x}^{3}=-\frac{3}{2}(\tau a)^{2} a_{x}^{3} .\right.
\end{gathered}
$$

Questa è la forma cercata, e si ha perciò l'importante equazione:

$$
u_{11} \tau_{22}-2 u_{12} \tau_{12}+u_{22} \tau_{11}=-\frac{2}{3} A j+i \alpha .
$$

Cerchiamo ora che cosa avvenga di questa equazione, se in luogo delle potenze delle $x$ vi si introducano, in ordine rovesciato e coi segni cambiati, $\mathrm{i}$ coefficienti della forma cubica:

$$
3\left(i_{1} j_{2}-j_{1} i_{2}\right)=3(i j) i_{x} j_{x}^{2} .
$$

11 primo membro diviene:

$$
3(a \tau)^{2}(i j)(i a)(j a)^{2}=-3(a \tau)(i a)(j a)^{2}\{(a i)(j \tau)-(a j)(\tau i)\}
$$

ovvero, omessa la parte moltiplicata per $(j a)^{3}$ :

$$
3(a \tau)(a i)^{2}(j a)^{2}(j \tau)=-6\left(j^{\prime} \tau\right)\left(j j^{\prime}\right)^{2}(j \tau)=-6\left(\tau \tau^{\prime}\right)^{2}=-6 G .
$$


Poi da $j$ si ricava:

$$
3(i j)\left(i j^{\prime}\right)\left(j j^{\prime}\right)^{2}=3(i \tau)^{2}=3 B \text {. }
$$

Finalmente la combinazione di $i \alpha$ colla funzione $3\left(i_{1} j_{2}-j_{1} i_{2}\right)$ dà:

$$
(i j)\left\{2\left(i i^{\prime}\right)\left(j i^{\prime}\right)(j \alpha)+\left(j i^{\prime}\right)^{2}(i \alpha)\right\} .
$$

La prima parte di questa espressione è identicamente nulla, perchè essa muta di segno se si scambia $j$ con $j^{\prime}$. La seconda è

$$
(i a)^{2}=2 M \text {. }
$$

Dunque, in seguito a queste operazioni, l'equazione proposta diviene:

$$
-6 C=-2 A B+2 M,
$$

onde si ottiene per $M$ il valore:

$$
M=A B-3 C,
$$

e questa è la seconda relazione fra gli invarianti.

\section{$\S 3$.}

Forme tipiche per $i, \tau, \$, j$.

Se si forma il determinante funzionale di $\alpha$ e di un covariante quadratico, ne risulta un nuovo covariante lineare. Siccome $\alpha$ è del $5^{\circ}$ ordine nei coefficienti di $u$, cosi le combinazioni di $\alpha$ con $i, \tau, \&$ danno covarianti lineari degli ordini 7, 5, 13. Fra essi l'ultimo ha una particolare importanza, ed è appropriato ad essere introdotto nelle forme tipiche, essendo la differenza del suo ordine con quello di a divisibile per 4; d'onde segue che, se si assumono $\alpha$ e:

$$
\gamma=\frac{1}{2}\left(\curvearrowright_{1} \alpha_{2}-\alpha_{1} \$_{2}\right)
$$

come variabili nella composizione delle forme tipiche, gli ordini dei coefficienti dei singoli termini saranno tutti numeri congrui rispetto al modulo 4 , e conseguentemente l'invariante del $18^{\circ}$ ordine, il quale è espresso irrazionalmente per mezzo degli altri non può entrare nei coefficienti. Invece esso d. il determinante della trasformazione, avendosi :

$$
\gamma_{1} \alpha_{2}-\alpha_{1} \gamma_{2}=R
$$


Prima di tutto esprimiamo $i, \tau$ e $\$$ per mezzo di $\alpha$ e $\gamma$. Dalle formole:

segue :

$$
\begin{aligned}
& \gamma=\gamma_{1} x_{1}+\gamma_{2} x_{2}, \\
& \alpha=\alpha_{1} x_{1}+\alpha_{2} x_{2}
\end{aligned}
$$

$$
\begin{aligned}
& \text { R. } x_{1}=\gamma \alpha_{2}-\gamma_{2} \alpha, \\
& \text { R. } x_{2}=-\gamma \alpha_{1}+\gamma_{1} \alpha,
\end{aligned}
$$

e per una qualsivoglia forma di grado $\nu$ :

$$
\phi=\left(\phi_{1} x_{1}+\phi_{2} x_{2}\right)^{2}
$$

si ottiene l'espressione simbolica:

$$
\begin{gathered}
R^{\nu} \phi=\left[\left(\phi_{1} \alpha_{2}-\phi_{2} \alpha_{1}\right) \gamma-\left(\phi_{1} \gamma_{2}-\phi_{2} \gamma_{1}\right) \alpha\right]^{\nu} \\
=\phi_{\alpha \nu} \cdot \gamma^{\nu}-\frac{\nu}{1} \cdot \phi_{\alpha^{\nu-1} \gamma} \cdot \gamma^{\nu-1} \alpha+\frac{\nu \cdot \nu-1}{1.2} \phi_{\alpha^{\nu-2} \gamma^{2}} \cdot \gamma^{\nu-2} \alpha^{2}+\cdots
\end{gathered}
$$

In particolare quindi sarà :

$$
\begin{aligned}
& R^{2} \cdot \$=\$_{\alpha \alpha} \cdot \gamma^{2}-2 \$_{\alpha \gamma} \cdot \alpha \gamma+\$_{\gamma \gamma} \cdot \alpha^{2}, \\
& R^{2} \cdot i=i_{\alpha \alpha} \cdot \gamma^{2}-2 i_{\alpha \gamma} \cdot a \gamma+i_{\gamma \gamma} \cdot \alpha^{2}, \\
& R^{2} \cdot \tau=\tau_{\alpha \alpha} \cdot \gamma^{2}-2 \tau_{\alpha \gamma} \cdot a \gamma+\tau_{y \gamma} \cdot \alpha^{y} .
\end{aligned}
$$

I coefficienti si determinano come segue. In primo luogo, per la definizione degli invarianti $R, M, N$, è :

$$
\$_{\alpha \alpha}=2 R, \quad i_{\alpha \alpha}=2 M, \quad \tau_{\alpha \alpha}=2 N \text {. }
$$

Poi, se $\phi$ esprime una qualunque delle funzioni $i, \tau$,, , si ha:

$$
\phi_{\alpha \gamma}=\frac{1}{2}\left|\begin{array}{ll}
\phi_{11} \alpha_{2}-\phi_{12} \alpha_{1} & \S_{11} \alpha_{2}-\$_{12} \alpha_{1} \\
\gamma_{12} \alpha_{2}-\phi_{22} \alpha_{1} & \delta_{12} \alpha_{2}-s_{22} \alpha_{1}
\end{array}\right|
$$

cioè $\phi_{u}$, è la metà del determinante funzionale di $\phi$ e $\mathcal{S}$, ove pongansi $\alpha_{i z}$, $-\alpha_{1}$ invece di $x_{1}, x_{2}$. Percio dalla espressione di questo determinante funzionale, superiormente data, si ha:

$$
s_{\alpha \gamma_{\gamma}}=0, i_{i \gamma}=-(A N-B M), \tau_{(y,}=-(B N-C M) .
$$


Le due combinazioni:

$$
S=A N-B M, \quad T=C M-B N,
$$

che sono le metà delle derivate di $-R^{2}$ rispetto ad $M$ ed $N$, sembrano abbastanza importanti per giustificare una particolare notazione. Per mezzo di questa abbiamo:

$$
\$_{\alpha \gamma}=0, \quad i_{\alpha \gamma}==-S, \quad \tau_{\alpha \gamma}=T .
$$

Da ultimo si trovano $\$_{\gamma \gamma}, i_{\gamma \gamma}, \tau_{\gamma \gamma}$, applicando le equazioni:

$$
\begin{aligned}
& \curvearrowright_{11} \vartheta_{22}-\$_{12}^{2}=A G-B^{2}, \\
& \curvearrowright_{11} i_{22}+\$_{22} i_{11}-2 \curvearrowright_{12} i_{12}=0 \\
& \curvearrowright_{11} \tau_{22}+\$_{22} \tau_{11}-2 \curvearrowright_{12} \tau_{12}=0
\end{aligned}
$$

alla forma tipica, onde esse diventano:

$$
\begin{aligned}
& \$_{\alpha \alpha} \Im_{\gamma \gamma}-\$_{\alpha \gamma}^{2}=R^{2}\left(A G-H^{2}\right)=4 N R^{2}, \\
& \$_{\alpha \alpha} i_{\gamma \gamma}-2 \$_{\alpha \gamma} i_{\alpha \gamma}+\$_{\gamma \gamma} i_{\alpha \alpha}=0, \\
& \$_{\alpha \alpha} \tau_{\gamma \gamma}-2 \$_{\alpha \gamma} \tau_{\alpha \gamma}+\$_{\gamma \gamma} \tau_{\alpha \alpha}=0 .
\end{aligned}
$$

Di qui si desume:

$$
s_{\gamma \gamma}=2 N R, \quad i_{\gamma \gamma}=-2 N M, \tau_{\gamma^{\prime}}=-2 N^{2},
$$

epperò le forme tipiche di $\curvearrowright, i$, sono le seguenti :

$$
\begin{aligned}
& 1_{2}^{1} R S=\gamma^{2}+N \alpha^{2}, \\
& { }_{2}^{1} R^{2} i=M\left(\gamma^{2}-N \alpha^{2}\right)+S \alpha \gamma, \\
& \frac{1}{2} R^{2} \tau=N\left(\gamma^{2}-N \alpha^{2}\right)-T \alpha \gamma .
\end{aligned}
$$

Queste equazioni danno:

$$
\begin{aligned}
\gamma^{2}+N \alpha^{2} & =\frac{R \&}{2}, \\
\gamma^{2}-N \alpha^{2} & =-\frac{S \tau+T i}{2}, \\
\alpha \gamma & =\frac{M \tau-N i}{2},
\end{aligned}
$$


dove si è adoperata soltanto la:

$$
M T+N S=-R^{2}
$$

Di qui si vede che $\alpha$ e $\gamma$ possono anche essere definiti come i fattori razionali della combinazione $M \tau-N i$. E finalmente dalle stesse equazioni segue:

$$
\begin{aligned}
N \alpha^{2} & =\frac{R S+T i+S \tau}{4}, \\
\gamma^{2} & =\frac{R S-T i-S \tau}{4}, \\
k \gamma & =\frac{M v-N i}{2} .
\end{aligned}
$$

Ora queste equazioni possono anzitutto essere utilizzate per determinare la forma tipica di $j$. Sebbene questa determinazione non sia necessaria per ciò che segue, tuttavia essa è degna d'attenzione per sè stessa, in quanto che serve già a mettere in chiaro il metodo generale di queste composizioni.

Posto, dietro le cose premesse:

$$
R^{2} \cdot j=j_{\alpha^{3} \cdot \gamma^{3}}-3 j_{\alpha^{2} \gamma} \cdot \gamma^{2} \alpha+3 j \alpha \gamma^{2} \cdot \gamma \alpha^{2}-j_{\gamma^{3}} \cdot \alpha^{3},
$$

si ha anche:

$$
\begin{aligned}
& R \cdot j_{\alpha \alpha}=R\left[j_{11} \alpha_{2}^{2}-2 j_{12} \alpha_{1} \alpha_{2}+j_{22} \alpha_{1}^{2}\right]=j_{\alpha^{3}} \cdot \gamma-j_{\alpha^{2} \gamma^{2}} \cdot \alpha, \\
& R \cdot j_{\alpha \gamma}=R\left[j_{11} \alpha_{22} \gamma_{2}-j_{12}\left(\alpha_{1} \gamma_{2}+\alpha_{2} \gamma_{1}\right)+j_{12} \alpha_{1} \gamma_{1}\right]=j_{\alpha^{2} \gamma} \cdot \gamma-j_{c \gamma^{2}} \cdot \alpha, \\
& R \cdot j_{\gamma \gamma}=R\left[j_{11} \gamma_{2}^{2}-2 j_{12} \gamma_{1} \gamma_{2}+j_{22} \gamma_{1}^{2}\right]=j \alpha \gamma^{2} \cdot \gamma-j_{\gamma^{3}} \cdot \alpha .
\end{aligned}
$$

Dunque se nelle formole superiori esprimenti $\alpha^{2}, \gamma^{2}, \alpha \gamma$, in luogo di $x_{1}^{2}$, $x_{1} x_{2}, x_{2}^{2}$ si pongono le quantità $j_{22},-j_{12}, j_{11}$, si otterrà :

$$
\begin{aligned}
& N\left(j_{\alpha^{3}} \cdot \gamma-j_{a^{2} \gamma} \cdot a\right)=\frac{R}{4}\left(R j_{y}+T j_{i}+S j_{l}\right), \\
& j_{\alpha \alpha^{2} \gamma} \cdot \gamma-j \alpha \gamma^{2} \cdot \alpha=\frac{l l}{2}\left(M_{j t}-N j_{i}\right), \\
& j_{\alpha, 2} \cdot \gamma-j \gamma^{3} \cdot \alpha=-\frac{R}{4}\left(R j_{\gamma}-T j_{i}-S j_{t}\right),
\end{aligned}
$$

dove si e posto per brevità: 


$$
\begin{aligned}
& j_{7}=\vartheta_{11} j_{22}-2 \curvearrowright_{12} j_{12}+\Im_{12} j_{11}, \\
& j_{i}=i_{11} j_{22}-2 i_{12} j_{12}+i_{22} j_{11}, \\
& j_{c}=\tau_{11} j_{22}-2 \tau_{12} j_{12}+\tau_{22} j_{11} .
\end{aligned}
$$

La seconda di queste funzioni è $2 \alpha$; la terza, come covariante lineare della forma cubica $j$, è identicamente nulla; la prima sia $\frac{p \Upsilon+q \alpha}{R}$. Allora le equazioni superiori, eguagliandn i coeficienti, danno:

$$
\begin{aligned}
& N_{j} \alpha^{3}=\frac{p R}{4}, \quad j_{\alpha^{2} \gamma}=0, \quad j_{\alpha \gamma^{2}}=\frac{p R}{4}, \\
& N j_{\alpha 2 \gamma}=-\left(\frac{q}{4}+\frac{T}{2}\right) R, \quad j_{\alpha \gamma^{2}}=N R, j_{\gamma^{3}}=-\left(\frac{q}{4}-\frac{T}{2}\right) R .
\end{aligned}
$$

Dal confronto di queste formole segue :

$$
\begin{aligned}
& q=-2 T, \quad p=4 N, \\
& j_{\alpha^{3}}=R, \quad j_{\alpha^{2} \gamma}=0, \quad j_{\alpha \gamma^{2}}=N R, \quad j_{\gamma^{3}}=T R,
\end{aligned}
$$

epperò la forma tipica di $j$ sarà :

$$
R^{2} \cdot j=\gamma^{3}+3 N \gamma \alpha^{2}-T \alpha^{3} .
$$

$\$ 4$.

\section{Rappresentazione di $u$.}

La rappresentazione di $u$ si ottiene in modo analogo a quella di $j$. A quest' ropo cominciamo dal formare le equazioni:

$$
\begin{array}{ll}
N^{2} \alpha^{4}=\frac{(R \S+\varphi)^{2}}{16}, & \alpha^{2} \gamma^{2}=\frac{\psi^{2}}{4}, \\
N \alpha^{3} \gamma=\frac{(R \curvearrowright+\varphi) \psi}{8}, & \alpha \gamma^{3}=\frac{(R S-\varphi) \psi}{8}, \\
N a^{2} \gamma^{2}=\frac{R^{2} \S^{2}-\psi^{2}}{16}, & \gamma^{4}=\frac{(R S-\varphi)^{2}}{16},
\end{array}
$$


dove per brevità si è posto:

$$
\phi=T i+S \tau, \quad \psi=M \tau-N i .
$$

Ora rappresentando la forma tipica di $u$ coll'equazione:

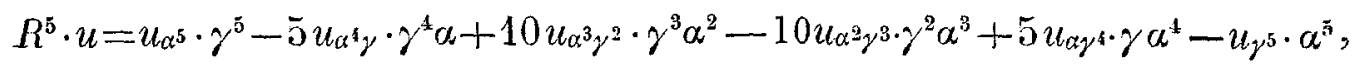
si ha di nuovo:

$$
\begin{aligned}
& u_{\alpha 5} \cdot \gamma-u_{\alpha^{8} \gamma} \cdot \alpha=R\left[\begin{array}{lll}
u_{111} \alpha_{2}^{4}-4 u_{1112} \alpha_{2}^{3} \alpha_{1} & +\cdots]
\end{array}\right] \\
& u_{\alpha^{4} \gamma} \cdot \gamma-u_{\alpha^{3} \gamma^{2}} \cdot \alpha=R\left[u_{1111} \alpha_{2}^{3} \gamma_{2}-u_{1112}\left(\alpha_{2}^{3} \gamma_{1}+3 \alpha_{1} \alpha_{2}^{2} \gamma_{2}\right)+\cdots\right] \text {, } \\
& u_{\alpha^{3} \gamma^{2}} \cdot \gamma-u_{\alpha^{2} \gamma^{3}} \cdot \alpha=R\left[u_{111} \alpha_{2}^{2} \gamma_{2}^{2}-2 u_{1112}\left(\alpha_{2}^{2} \gamma_{1} \gamma_{2}+\gamma_{2}^{2} \alpha_{1} \alpha_{2}\right)+\cdots\right] \text {, } \\
& u_{\alpha^{2} \gamma^{3}} \cdot \gamma-u_{\alpha \gamma^{4}} \cdot \alpha=R\left[u_{111} \alpha_{2} \gamma_{2}^{3}-u_{1112}\left(\alpha_{1} \gamma_{2}^{3}+3 \alpha_{2} \gamma_{1} \gamma_{2}^{2}\right)+\cdots\right] \text {, } \\
& u_{\alpha \gamma^{4}} \cdot \gamma-u_{y^{5}} \cdot \alpha=R\left[u_{111} \gamma_{2}^{4}-u_{112} \gamma_{2}^{3} \gamma_{1}+\cdots\right] \text {; }
\end{aligned}
$$

i termini fra parentesi nel secondo membro si ottengono surrogando le potenze delle $x$ in $\alpha^{4}, \alpha^{3} \gamma$, ecc., colle quantitâ $u_{1111},-u_{1112}, \ldots$ Introducendo le notazioni:

$$
\begin{gathered}
u_{\varphi}=u_{11} \phi_{22}-2 u_{12} \phi_{12}+u_{22} \hat{\phi}_{11}, \text { ecc. } \\
u_{\varphi \psi}=u_{\psi \varphi}=\left(u_{\varphi}\right)_{11} \psi_{22}-2\left(u_{\varphi}\right)_{12} \psi_{12}+\left(u_{\varphi}\right)_{22} \psi_{11}, \text { ecc. }
\end{gathered}
$$

si hanno, per la determinazione dei coefficienti della forma tipica, le equazioni seguenti:

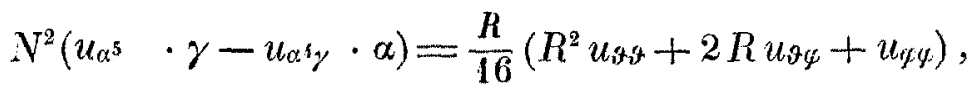

$$
\begin{aligned}
& N\left(u_{\alpha^{4} \gamma} \cdot \gamma-u_{\left(\alpha^{3} \gamma^{2}\right.} \cdot \alpha\right)=\frac{n}{8}\left(R u_{q \psi}+u_{q y b}\right), \\
& N\left(u_{\sigma^{3} \gamma^{2}} \cdot \gamma-u_{c^{2} \gamma^{3}} \cdot \alpha\right)=\frac{n}{16}\left(R^{2} u_{9,9}-u_{\psi \varphi}\right), \\
& \imath_{\alpha} \alpha^{3} \gamma^{2} \cdot \gamma-u_{\alpha^{2}} \gamma^{3} \cdot \alpha=\frac{R}{4} u \psi \psi, \\
& u_{\alpha^{2} \gamma^{8}} \cdot \gamma-u_{\alpha \gamma^{4}} \cdot \alpha=\frac{R}{8}\left(R u_{\S \psi}-u_{\psi \psi}\right), \\
& u_{\alpha \gamma^{4}} \cdot \gamma-u_{\gamma} \cdot \alpha=\frac{R}{16}\left(R^{2} u_{\vartheta \vartheta}-2 R u_{\vartheta}+u_{y y}\right) \cdot
\end{aligned}
$$


Il confronto della terza colla quarta equazione dà:

$$
R^{2} u_{\vartheta \vartheta}=u_{\mathscr{Y} \psi}+4 N u_{\psi}, \psi
$$

onde le sei equazioni precedenti possono essere sostituite dalle cinque seguenti :

$$
\begin{aligned}
& N^{2}\left(u_{\alpha^{5}} \cdot \gamma-u_{\alpha^{4} \gamma} \cdot \alpha\right)=\frac{R}{8}\left(2 N u_{\psi \psi}+u_{\mathscr{\varphi} \varphi}+R u_{\vartheta \Psi}\right), \\
& N\left(u_{\alpha^{4} \gamma} \cdot \gamma-u_{\alpha^{3} y^{2}} \cdot \alpha\right)=\frac{R}{8}\left(R u_{\Im \psi}+u_{y \psi}\right),
\end{aligned}
$$

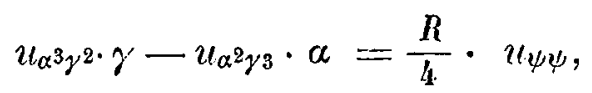

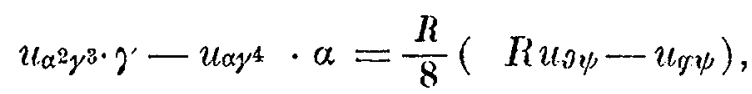

$$
\begin{aligned}
& u \alpha \gamma^{4} \cdot \gamma-u_{\gamma^{5}} \cdot a=\frac{R}{8}\left(2 N u_{\psi \psi}+u_{\digamma \psi}-R u_{\% \varphi}\right) .
\end{aligned}
$$

Dei cinque covarianti lineari :

$$
\begin{aligned}
& u_{\psi \psi}=a_{\psi / \mu \psi} \gamma-b_{\psi \psi} \alpha, \quad u_{\varphi \psi \psi}=a_{\varphi \psi} \gamma-b_{y, \psi} \alpha, \quad u_{\varphi \psi}=a_{\psi \varphi} \gamma-b_{\varphi \varphi} \alpha, \\
& R u_{\vartheta \psi}=a_{\vartheta \psi} \gamma-b_{\vartheta q} \alpha, \quad R u_{\vartheta \psi}=a_{\vartheta \psi} \gamma \gamma-b_{\vartheta \psi} \alpha
\end{aligned}
$$

due possono essere espressi mediante gli altri tre. In fatti il confronto dei coefficienti nelle cinque equazioni superiori dà:

$$
\begin{aligned}
& N^{2} u_{\alpha 5}=\frac{R}{8}\left(2 N^{\dagger} a_{\psi \psi}+a_{\varphi \varphi}+a_{\vartheta \varphi}\right), \quad N^{2} u_{\alpha^{4}} y=\frac{R}{8}\left(2 N b_{\psi^{2} \psi}+b_{\varphi \varphi}+b_{\vartheta \varphi}\right), \\
& N u_{\alpha^{4} \gamma}=\frac{R}{8}\left(\alpha_{\vartheta \psi} \psi+a_{\psi \psi}\right), \quad N u_{\alpha^{3} \gamma^{2}}=\frac{R}{8}\left(b_{\vartheta \psi}+b_{\xi \psi}\right), \\
& u_{\alpha^{3} \gamma^{2}}=\frac{R}{4} a_{\psi \psi}, \quad \quad u_{\alpha^{2}, \gamma^{3}}=\frac{R}{4} b_{\psi \psi}, \\
& u_{\alpha}^{2} \gamma^{3}=\frac{R}{8}\left(a_{\vartheta \psi}-a_{\psi \psi \psi}\right), \quad u_{\alpha \gamma^{\prime}}=\frac{R}{8}\left(b_{\vartheta \psi}-b_{\varphi \psi}\right),
\end{aligned}
$$

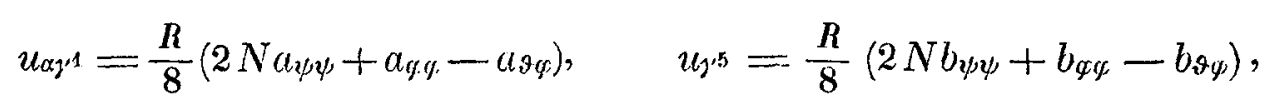


Si ha per conseguenza:

$$
\begin{aligned}
2 N b_{\psi \psi}+b_{\varphi \varphi}+b_{\vartheta \varphi} & =N\left(a_{\vartheta \psi}+a_{\varphi \psi}\right), \\
b_{\vartheta \psi}+b_{\varphi \psi} & =2 N a_{\psi \psi}, \\
2 b_{\psi \psi} & =a_{\vartheta \psi}-a_{\varphi \psi}, \\
b_{\vartheta \psi}-b_{\varphi \psi} & =2 N a_{\psi \psi}+a_{\varphi \varphi}-a_{\vartheta \varphi} .
\end{aligned}
$$

Queste equazioni forniscono i valori:

$$
\begin{array}{ll}
a_{\vartheta \psi}=2 b_{\psi \psi}+a_{\varphi \psi}, & a_{\vartheta \varphi}=2 b_{\varphi \psi}+a_{\varphi \psi}, \\
b_{\vartheta \psi}=2 N a_{\psi \psi}-b_{\varphi \psi}, & b_{\vartheta \varphi}=2 N a_{\varphi \psi}-b_{\varphi \varphi},
\end{array}
$$

introducendo i quali si hanno pei richiesti coefficienti le espressioni :

$$
\begin{aligned}
N u_{\alpha^{5}}^{2} & =\frac{R}{4}\left(N a_{\psi \psi}+a_{\varphi \varphi}+b_{\varphi \psi}\right), \\
N u_{\alpha^{4} \gamma} & =\frac{R}{4}\left(b_{\psi \psi}+a_{\psi \psi}\right), \\
u_{\alpha^{3} \gamma^{2}} & =\frac{R}{4} a_{\psi \psi} \cdot \\
u_{\alpha^{2} \gamma^{3}} & =\frac{R}{4} b_{\psi \psi}, \\
u_{\alpha \gamma^{4}} & =\frac{R}{4}\left(N a_{\psi \psi}-b_{\varphi \psi \psi}\right), \\
u_{\gamma^{5}} & =\frac{R}{4}\left(N b_{\psi \psi}+b_{\varphi \varphi}-N a_{\varphi \psi}\right) .
\end{aligned}
$$

Finalmente rimane ad esprimere i covarianti $u_{\psi \psi}, u_{\psi \psi}, u_{\psi \psi}$ per mezzo di $\alpha$ e $\gamma$. Per la definizione di $\phi$ e $\psi$ si ha:

$$
\begin{aligned}
& u_{\varphi \varphi}=T^{2} u_{i i}+2 S T u_{\imath t}+S^{2} u_{\tau t}, \\
& u_{\varphi \psi \psi}=-N T u_{i i}+(M T-N S) u_{\imath \tau}+M S u_{t \tau}, \\
& u_{\psi \psi}=N^{2} u_{i i}-\quad 2 M N u_{\imath \tau}+M^{2} u_{\tau \tau} .
\end{aligned}
$$

Inoltre, per le cose precedenti, è :

$$
u_{i}=-2 j, \quad u_{\tau}=-\frac{2 d j}{3}+i \alpha .
$$


dunque :

$$
\begin{gathered}
u_{i i}=-2 j_{i}=-4 \alpha, \quad u_{i \tau}=-2 j_{\tau}=0, \\
\left.\left.u_{\tau \tau}=\frac{2}{3}\right\} B \alpha+i_{1} \alpha_{1} \tau_{22}-\left(i_{1} \alpha_{2}+\alpha_{1} i_{2}\right) \tau_{12}+i_{2} \alpha_{2} \tau_{11}\right\} .
\end{gathered}
$$

L'espressione che segue dopo $B \alpha$ fra le parentesi ha la forma simbolica $i_{x}(i \tau)(\alpha \tau)$. Ora è evidente che:

dunque :

$$
\begin{aligned}
& i_{x}(i \tau)(\alpha \tau)+\tau_{x}(i \tau)(\alpha i)=2 \mathcal{A}_{x}(\alpha)=-4 \gamma \\
& i_{x}(i \tau)(\alpha \tau)-\tau_{x}(i \tau)(\alpha i)=\alpha_{x}(i \tau)^{2}=2 B \alpha
\end{aligned}
$$

e :

$$
i_{x}(i \tau)(\alpha \tau)=B \alpha-2 \gamma
$$

$$
u_{\tau t}=\frac{4}{3}(B \alpha-\gamma)
$$

Quindi anche:

ossia :

$$
\begin{aligned}
& u_{\varphi \varphi}=-4 \alpha T^{2}+\frac{4}{3}(B \alpha-\gamma) S^{2}, \\
& u_{\varphi \psi}=4 \alpha N T+\frac{4}{3}(B \alpha-\gamma) M S, \\
& u_{\psi \psi}=-4 \alpha N^{2}+\frac{4}{3}(B \alpha-\gamma) M^{2},
\end{aligned}
$$

$$
\begin{array}{lll}
a_{\varphi \psi}=-\frac{4}{3} S^{2}, & a_{\varphi \psi}=-\frac{4}{3} M S, & a_{\psi \psi}=-\frac{4}{3} M^{2}, \\
b_{\varphi \varphi}=4 T^{2}-\frac{4}{3} B S^{2}, & b_{\varphi \psi}=-4 N T-\frac{4}{3} \cdot B M S, & b_{\psi \psi}=4 N^{2}-\frac{4}{3} B M^{2} .
\end{array}
$$

Introducendo questi valori, è facile effettuare la divisione per $N$ e per $N^{2}$ nei primi due coefficienti di $u$. Cioè sarà:

$$
\begin{aligned}
u_{\alpha^{4} \gamma} & =R\left(N-\frac{M}{3} \cdot \frac{B M+S}{N}\right)=R\left(N-\frac{A M}{3}\right) \\
u_{\alpha^{5}} & =\frac{R}{N^{2}}\left(-\frac{N M^{2}}{3}-\frac{S^{2}}{3}-N T-\frac{B M S}{3}\right)=-\frac{R}{3 N}\left(M^{2}+A S+3 T\right) \\
& =-\frac{R}{3 N}\left\{M\left(M-A B+3 C^{\prime}\right)+N\left(A^{2}-3 B\right)\right\}=R\left(B-\frac{A^{2}}{3}\right) .
\end{aligned}
$$

Annali di Matematica. 
Per conseguenza i coefficienti di $u$ sono:

$$
\begin{array}{ll}
u_{\alpha^{5}}=R\left(B-\frac{A^{2}}{3}\right), & u_{\alpha^{4} \gamma}=R\left(N-\frac{A M}{3}\right), \\
u_{\alpha^{3} \gamma^{2}}=-\frac{R M^{2}}{3}, & u_{\alpha^{2} \gamma^{3}}=R\left(N^{2}-\frac{B M^{2}}{3}\right), \\
u_{\alpha \gamma^{4}}=R\left(N T-\frac{M N-B S}{3} M\right), u_{\gamma^{5}}=R\left(N^{3}+T^{2}+\frac{N M S-B M^{2} N-B S^{2}}{3}\right),
\end{array}
$$

e la forma tipica di $u$ sarà :

$$
\begin{aligned}
R^{4} \cdot u & =\left(B-\frac{A^{2}}{3}\right) \gamma^{5}-5\left(N-\frac{A M}{3}\right) \gamma^{4} \alpha-10 \frac{M^{2}}{3} \gamma^{3} \alpha^{2}-10\left(N^{2}-\frac{B M^{2}}{3}\right) \gamma^{2} \alpha^{3}+ \\
& +5\left(N T-\frac{M N-B S}{3} M\right) \gamma \alpha^{4}-\left(N^{3}+T^{2}+\frac{N M S-B M^{2} N-B S^{2}}{3}\right) \alpha^{5}
\end{aligned}
$$

$\$ 5$.

Caso eccezionale : $R=0$. Seconda forma tipica.

La rappresentazione tipica di $u$ che precede è sempre possibile, purchè $R$ non sia zero. Se $R$ è zero, per ottenere la forma tipica, bisogna invece di $\gamma$ scegliere un altro covariante. Tale è il seguente derivato da $i$ :

$$
\beta=\frac{1}{2}\left(i_{1} \alpha_{2}-\alpha_{1} i_{2}\right)
$$

ed il determinante della trasformazione è in questo caso:

$$
\beta_{1} \alpha_{2}-\alpha_{1} \beta_{2}=M
$$

La forma tipica di $u$ sarè allora:

$$
M^{5} \cdot u=u_{\alpha^{5}} \cdot \beta^{5}-5 u_{\alpha \alpha^{4} \beta} \cdot \beta^{4} \alpha+10 u_{\alpha^{3} \beta^{2}} \cdot \beta^{z} \alpha^{2}-10 u_{\alpha^{2} \beta^{3}} \cdot \beta^{2} \alpha^{3}+5 u_{\alpha \beta \beta} \cdot \beta \alpha^{4}-u_{3,3} \cdot \alpha^{5} \cdot
$$

Noi la svilupperemo in generale, senza supporre da principio la condizions $R=0$. Per convincersi che questa condizione semplificherà molto l'espressione di $M^{5} u$, basta enumerare le dimensioni; donde segue che $u_{\alpha^{5}}, u_{\alpha^{3} \beta^{2}}, u_{\alpha \beta^{4}}$ contengono il fattore $R$, eppero si annullano con esso; cioè se $R=0, \alpha$ è un fattore di $u$, ed il rimanente fattore di quarto grado non 
contiene che $\mathrm{j}$ quadrati di $\alpha$ e $\beta$. Dunque l'equazione $u=0$ ha allora un fattore razionale conosciuto $\alpha$, e l' equazione $\frac{u}{\alpha}=0$ è risolubile mediante radici quadrate.

Per cercare, indipendentemente dalla condizione $R=0$, l'espressione tipica di $M^{5} u$, mediante $\alpha$ e $\beta$, noi possiamo tenere precisamente la stessa strada seguita dianzi, così che non sarà necessario di indicare di nuovo completamente tutti i passaggi. Da prima si trova:

$$
\begin{aligned}
& \frac{M i}{2}=\beta^{2}+\frac{A}{4} \alpha^{2}, \\
& \frac{M^{2} \tau}{2}=N \beta^{2}+R \alpha \beta+\left(\frac{M B}{2}-\frac{A N}{4}\right) \alpha^{2}, \\
& \frac{M^{2} \Omega}{2}=R \beta^{2}-S \alpha \beta+\frac{A R}{4} \alpha^{2} .
\end{aligned}
$$

La prima di queste equazioni c'insegna che questa forma tipica ha una assai semplice connessione con quella del sig. Hermers. In fatti le variabili introdotte dal sig. Hermite sono $\mathrm{i}$ fattori di $i$, cioè :

$$
\beta+\frac{\alpha}{2} \sqrt{-1}, \quad \beta-\frac{\alpha}{2} \sqrt{-1} .
$$

Passando poi a comporre $j$, troviamo:

$$
M^{3} j=R \beta^{3}-\frac{3}{4} S \beta^{2} \alpha-\frac{3 A R}{4} \beta \alpha^{2}+\left(M^{2}+\frac{\Lambda S}{8}\right) \alpha^{3}
$$

E da ultimo pei coefficienti della forma tipica di $u$ si ottiene la serie seguente:

$$
\begin{aligned}
& u_{\alpha^{5}}=M\left(B-\frac{A^{2}}{3}\right), \\
& u_{\alpha^{4} \beta}=M\left(N-\frac{A M}{3}\right)+\frac{S}{2}\left(B-\frac{A^{2}}{3}\right), \\
& u_{\alpha^{3} \beta^{2}}=-R\left(M+\frac{A}{4}\left(B-\frac{A^{2}}{3}\right)\right), \\
& u_{\alpha^{2} \beta^{3}}=M\left(\frac{A^{2} M}{12}-\frac{A N}{4}-\frac{S}{2}\right)-\frac{A S}{8}\left(B-\frac{A^{2}}{3}\right),
\end{aligned}
$$




$$
\begin{aligned}
& u_{\alpha \beta^{4}}=R\left(\frac{M A}{2}+\frac{A^{2}}{16}\left(B-\frac{A^{2}}{3}\right)\right) \\
& u_{\beta^{5}}=M\left(\frac{A^{2} N}{16}-\frac{A^{3} M}{48}+\frac{A S}{4}+M^{2}\right)+\frac{A^{2} S}{32}\left(B-\frac{A^{2}}{3}\right),
\end{aligned}
$$

In particolare, per $R=0, u$ ha la rappresentazione seguente:

$$
\begin{aligned}
M^{5} u= & -5 \beta^{4} \alpha\left[M\left(N-\frac{A M}{3}\right)+\frac{S}{2}\left(B-\frac{A^{2}}{3}\right)\right] \\
& -10 \beta^{2} \alpha^{3}\left[M\left(\frac{A^{2} M}{12}-\frac{A N}{4}-\frac{S}{2}\right)-\frac{A S}{8}\left(B-\frac{A^{2}}{3}\right)\right] \\
& -\alpha^{5}\left[M\left(\frac{A^{2} N}{16}-\frac{A^{3} M}{48}+\frac{A S}{4}+M^{2}\right)+\frac{A^{2} S}{32}\left(B-\frac{A^{2}}{3}\right)\right] .
\end{aligned}
$$

$\$ 6$.

Secondo caso d'eccezione: $R=0, M=0$.

Nessuna delle precedenti trasformazioni è possibile quando $R$ ed $M$ sono nulli simultaneamente. Per la discussione di questi casi, supponiamo da prima che nessuno dei covarianti $\alpha, \tau, j, i$ sia identicamente zero .

Siccome per $M=0, R$ si riduce alla forma semplice $A N^{\prime}$, così dobbiamo cominciare dal distinguere due casi, secondo che è zero $A$ od $N$, ed invero da prima uno solo di questi invarianti.

1. Se $M=0, A=0$, l'espressione di $M$ dà anche $C=0$; invece supporremo che $B$ sia diverso da zero, affinchè non si annulli anche $N$. In questo caso $i$ e $\tau$ sono quadrati, però fra loro differenti; siccome inoltre $M=i_{11} \alpha_{2}^{2}-2 i_{12} \alpha_{1} \alpha_{2}$ $+i_{22} \alpha_{1}^{2}$ mostra che $i$ è divisibile per $\alpha$, cosi ne segue:

$$
i=\rho \cdot \alpha^{2}, \tau=\sigma \cdot \eta^{2}
$$

dove $\eta$ è un' espressione lineare. Essendo 'poi $\tau$ il determinante di $j$, anche $j$ conterrà $\eta$ come doppio fattore, cioè sarà:

$$
j=n^{2}(3 p \alpha+q n)
$$


Se ora si considerano $\alpha$ ed $\eta$ come variabili, il modo col quale $j$ è generato mediante $i$ ed $u$, dà per quest' ultima forma la condizione che $\frac{\partial^{2} u}{\partial \eta^{2}}$ sia proporzionale a $j$; dunque:

$$
u=p^{\prime} a^{5}+5 q^{\prime} a^{4} \eta+5 \eta^{\prime} a n^{4}+s^{\prime} \eta^{5}
$$

Ricavando di qui $i$ e ponendolo proporzionale ad $\alpha^{2}$, si trova :

$$
\frac{1}{2} \rho \alpha^{2}=p^{\prime} r^{\prime} \cdot \alpha^{2}+\left(p^{\prime} s^{\prime}-3 q^{\prime} r^{\prime}\right) \alpha \eta+q^{\prime} s^{\prime} \cdot \eta^{2} \text {. }
$$

I coefficienti $p^{\prime}$ ed $r^{\prime}$ non possono essere nulli, dunque $s^{\prime}=0, q^{\prime}=0$. Cioè in questo caso è :

$$
u=\alpha\left(p^{\prime} \alpha^{4}+r^{\prime} \eta^{4}\right)
$$

2. Se $M=0, N=0$, $i$ e $\tau$ hanno il fattor comune $\alpha$; e fra gli invarianti sussistono le equazioni:

$$
A B=3 G, \quad A C=B^{2},
$$

ciò̀ $B=0, C=0$, oppure $C=\frac{A^{3}}{9}$ e $B=\frac{A^{2}}{3}$.

L'invariante $A$ si suppone diverso da zero, per non ricadere nel caso antecedente.

1) $B=0, C=0$. Qui $\tau$ è un quadrato, $\tau=\rho . \alpha^{2}$, ed $i$ ha il fattore $\alpha$ ed un altro fattore diverso da $\alpha$, cioè $i=\sigma . \alpha \eta$. Quindi $j$ è della forma :

$$
j=\alpha^{2}(p \alpha+3 q \eta) \text {. }
$$

Allora l' equazione che definisce $j$ per mezzo di $u$ ed $i$, mostra che $\frac{\partial^{2} u}{\partial \alpha \partial n}$ è proporzionale a $j$, epperó $u$ avrà la forma:

$$
u=p^{\prime} \alpha^{5}+5 q^{\prime} \alpha^{4} \eta+10 r^{\prime} \alpha^{3} \eta^{2}+s^{\prime} \eta^{5}
$$

Di qui si ricava per $i$ l'espressione :

$$
\left(p^{\prime} \alpha+q^{\prime} \eta\right) s^{\prime} \eta+3 q^{\prime} \alpha^{2},
$$


dunque $r^{\prime}$ e $q^{\prime}$ devono essere nulli. Per tal modo si ha finalmente per $u$ la forma :

$$
u=p^{\prime} \alpha^{5}+s^{\prime} \eta^{5}
$$

Ma allora $j=0$; dunque l'annullarsi di $B$ e $C$ trae necessariamente seco che vi sia qualche covariante identicamente nullo.

2) $C=\frac{A^{3}}{9}, B=\frac{A^{2}}{3}$. In questo caso, se $\alpha$ non si annulla, $\tau$ ed $i$ sono divisibili per $\alpha$, ma nessuno di essi è un quadrato.

Posto:

$$
\tau=2 \rho . \alpha_{\eta}, \quad i=\sigma \cdot \alpha^{2}+2 \pi \cdot \alpha_{\eta},
$$

sarà :

$$
j=p \alpha^{3}+q \eta^{3}
$$

e se ora da $i$ e $j$ si deriva $\alpha$, si ottiene $\sigma q \eta$, che in nessun modo può coincidere con $a$ stesso. Dunque è effettivamente impossibile che $i$ e $\tau$ siano divisibili per $\alpha$, senza che $\alpha$ si annulli identicamente: il qual caso sarà esaminato meglio in seguito.

Resta da indagare il caso che si verifichino simultaneamente le ipotesi 1. 2., cioè che $A, B, C$ siano tutti e tre eguali a zero. Allora $i$ e $\tau$ sono quadrati non differenti fra loro che per un fattore costante, se pure uno di essi non si annulla. Supposto ancora che $\alpha$ non sia zero, dovrà essere $i=\rho \alpha^{2}, \tau=\sigma \alpha^{2}$, epperò :

$$
j=p \alpha^{3}+3 q \alpha^{2} n
$$

e $\frac{\partial^{2} u}{\partial n^{2}}$ proporzionale a $j$, onde:

$$
u=p^{\prime} \alpha^{5}+5 q^{\prime} a^{4} n+10 r^{\prime} a^{3} \eta^{2}+10 s^{\prime} \alpha^{2} \eta^{3}
$$

Formando $i$, risulta l' espressione :

$$
-4\left(q^{\prime} \alpha+r^{\prime} \eta\right) s^{\prime} \alpha+3\left(r^{\prime} \alpha+s^{\prime} \eta\right)^{2}
$$

ed affinchè questa sia proporzionale ad $a^{2}$, dev' essere $s^{\prime}=0$; ma allora in $j$ sarebbe anche $q=0$, e per conseguenza $\tau$ identicamente nullo: caso che sarà trattato più tardi. 
$\$ \%$.

Casi eccezionali che risnltano dall' annullarsi di covarianti.

Studiamo da prima il caso che si annulli $\alpha$. Allora $M, N, R$ sono eguali a zero. Cominciamo col supporre che $\tau$ non sia nullo.

1. Non sia $i$ un quadrato, ma invece $i=2 \rho x y$. A cagione di $\alpha$ nullo è:

epperò :

$$
\frac{\partial^{2} j}{\partial x \partial y}=0
$$

$$
j=p x^{3}+q y^{3}, \quad \tau=2 \sigma x y
$$

cioè $\tau$ proporzionale ad $i$. Siccome poi anche $\frac{\partial^{2} u}{\partial x \partial y}$ sarà proporzionale a $j$, così :

$$
u=p^{\prime} x^{5}+b q^{\prime} x^{4} y+5 r^{\prime} x y^{4}+s^{\prime} y^{4}
$$

Di qui si ricava per $i$ la forma:

$$
\left(p^{\prime} x+q^{\prime} y\right)\left(r^{\prime} x+s^{\prime} y\right)-4 q^{\prime} r^{\prime} x y,
$$

eppero dev' essere $p^{\prime} r^{\prime}=q^{\prime} s^{\prime}=0$, senza che si annulli $p^{\prime} s^{\prime}-3 q^{\prime} r^{\prime}$; il che non puó aver luogo se non sono zero $p^{\prime}$ ed $s^{\prime}$, ovvero $q^{\prime}$ ed $r^{\prime}$. Ma nel secondo caso sarebbe nullo $j$; dunque dee sussistere il primo, e si avrà :

$$
u=5 x y\left(q^{\prime} x^{3}+r^{\prime} y^{3}\right)
$$

Dunque, se $\alpha$ è zero senza che lo sia anche $\tau$. e senza che $i$ sia un quadrato, $u$ è il prodotto di una forma cubica pel suo determinante.

2. Se $i$ e un quadrato, $i=\rho x^{2}$, si ha $A=0$, epperò anche $B=0, C=0$, a cagione di $M=0, N=0$; donde segue che se $\tau$ non è nullo, sarà $\tau=\sigma x^{2}$. Quindi $j=3 p x^{2} y$, e per conseguenza, siccome $\frac{\partial^{2} u}{\partial y^{2}}$ i proporzionale a $j$ :

$$
u=p^{\prime} x^{5}+5 q^{\prime} x^{4} y+10 r^{\prime} x^{2} y^{3}
$$


Formando $i$, otteniamo l' espressione:

$$
-4 q^{\prime} r^{\prime} x^{2}+3 r^{\prime 2} y^{2}
$$

la quale, per coincidere con $i$, richiede $r^{\prime}=0$; ma allora si annulla $i$ : caso che dev' essere trattato più avanti.

Passiamo ora ai casi nei quali si annulla $\tau$, essendo $\alpha$ e $j$ differenti da zero. Allora $j=\rho x^{3}$ e, siccome $\alpha$ sarà proporzionale a $\frac{\partial^{2} j}{\partial x^{2}}, \operatorname{così~} \alpha=\sigma x$. Dalla relazione identica fra $u$ e $j$, segue $u_{222}=0$, dunque:

$$
u=p x^{5}+5 q x^{4} y+10 r x^{3} y^{2} \text {. }
$$

Formando $i=2 \cdot 3 r^{2} x^{2}$ e combinandolo con $u$, si ottiene per $j$ un'espressione che coincide colla precedente. Ma la combinazione di $i$ con $j$ fornisce $\alpha=0$. Dunque, se $\tau$ è nullo, si annulla necessariamente anche $\alpha$, ed in questo caso $u$ ha un fattore triplo.

Se $j=0$, si annullano anche $\tau, \alpha, B, C$. Supposto che $i$ non sia zero, distinguiamo due casi .

1. Se $i$ non è un quadrato, sia $i=2 \rho x y$. Allora sarà $\frac{\partial^{2} u}{\partial x \partial y}=0$, epperò $u \grave{\partial}$ la somma di due quinte potenze.

2. Se $i$ è un quadrato, $i=\rho x^{2}$, si ha $\frac{\hat{\sigma}^{2} u}{\partial y^{2}}=0$, epperò :

$$
u=p x^{\mathrm{x}}+5 q x^{4} y \text {. }
$$

Ma allora si annulla contemporaneamente $i$.

Non resta dunque da esaminare che il caso dell'annullarsi di $i$, che trae seco anche $j=0$, ecc.

Questo caso ha primieramente luogo se $u$ è una quinta potenza. Supposto che ciò non sia, potremo scegliere per variabili due fattori differenti di $u$ e porre:

$$
\imath=\vdots b x^{4} y+10 c x^{3} y^{2}+10 d x^{2} y^{3}+5 e x y^{4}
$$

L' annullamento di $i$ esige le eguaglianze:

$$
3 c^{2}-4 b d=0, \quad 2 c d-3 b e=0, \quad 3 d^{2}-4 c e=0 \text {. }
$$


Allora 0 è zero $b$ e quindi anche $c$ e $d$; ovvero sono zero $e, d, c$; ed in entramti i casi $u$ contiene un fattore quadruplo. Da ultimo si può prendere :

$$
d=\frac{3 c^{2}}{4 b}, \quad e=\frac{27 c^{3}}{6 ! b^{2}}
$$

ma questo non si può accordare colla seconda delle equazioni superiori.

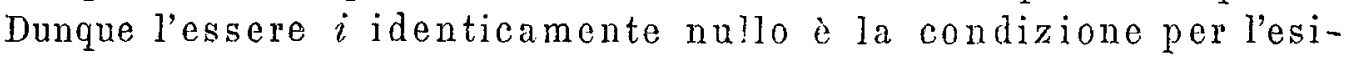
stenza di una radice quadrupla in $u=0$.

$\$ 8$.

Forme di sesto gnado. Fon me fondancentali-

Indichiamo una data forma binaria di sesto grado con:

$$
u=a x_{1}^{6}+6 b x_{1}^{3} x_{2}+15 c x_{1}^{4} x_{2}^{2}+20 d x_{1}^{3} x_{2}^{3}+15 e x_{1}^{0} x_{2}^{4}+6 f x_{1} x_{2}^{3}+g x_{2}^{6} .
$$

Se, conservando la notazione già adoperata, ricaviamo da essa la forma:

$$
u_{1111} y_{1}^{4}+4 u_{1112} y_{1}^{3} y_{2}+6 u_{1122} y_{1}^{2} y_{2}^{2}+4 u_{1222} y_{1} y_{2}^{3}+u_{2222} y_{2}^{4}
$$

che è del quarto grado rispetto alle $y$ e del secondo rispetto alle $x$; e se prendiamo il primo invariante di questa forma rispetto alle $y$, otteniamo una forma di quarto grado in $x$ :

$$
\begin{aligned}
k & =6\left(u_{1111} u_{2222}-4 u_{112} u_{1222}+3 u_{1122}^{2}\right) \\
& =\alpha x_{1}^{4}+4 \beta x_{1}^{3} x_{2}+6 \gamma x_{1}^{2} x_{2}^{2}+4 \delta x_{1} x_{2}^{3}+\varepsilon x_{2}^{4},
\end{aligned}
$$

dove $\mathrm{i}$ singoli coefficienti hanno le significazioni seguenti:

$$
\begin{aligned}
& \alpha=6\left(a e-4 b d+3 c^{2}\right), \\
& \beta=3(a f-3 b e+2 c d), \\
& \gamma=a g-9 c e+8 d^{2}, \\
& \delta=3(b g-3 c f+2 d e), \\
& \therefore=6\left(c g-4 d f+3 e^{2}\right) .
\end{aligned}
$$


Questa forma $k$ è di fondamentale importanza per la teoria delle forme di sesto grado. Ponendo simbolicamente:

$$
u=\left(a_{1} x_{1}+a_{2} x_{2}\right)^{6}=a_{x}^{2}=a_{x}^{\prime 2}=a_{x}^{\prime \prime 2} \ldots
$$

si ottieno per $k$ la rappresentazione simbolica:

$$
k=3\left(a, a^{\prime}\right)^{4} a_{x .0}^{2} a_{x}^{\prime 2}
$$

Fra $u$ e $k$ ha luogo una notevole relazione che è espressa dall'annullarsi del covariante di quarto grado formato con esse:

$$
h=u_{111} k_{222}-3 u_{112} k_{122}+3 u_{122} k_{112}-u_{222} k_{111} .
$$

In fatti dalla rappresentazione simbolica di $k$ si ha:

$$
k_{111} y_{1}^{3}+3 k_{112} y_{1}^{2} y_{2}+3 k_{122} y_{1} y_{2}^{2}+k_{222} y_{2}^{3}=3\left(a, c^{\prime}\right)^{4} a^{\prime}{ }_{x} a_{y}^{\prime} a_{y}^{2}
$$

e quindi, sostituendo alle quantità $y_{1}^{3}, y_{1}^{2} y_{2}, y_{1} y_{2}^{2}, y_{2}^{3}$ ordinatamente le:

$$
-u_{222}=-a_{2}^{\prime \prime 3} \cdot a_{x}^{\prime \prime 3}, u_{122}=a_{2}^{\prime \prime 2} a_{1}^{\prime \prime} \cdot a_{x}^{\prime 3},-u_{12}=-a_{2}^{\prime \prime} a_{1}^{\prime \prime 2} \cdot a_{x}^{\prime \prime 3}, u_{111}=a_{1}^{\prime \prime 3} \cdot a_{x}^{\prime \prime 3},
$$

sarà :

$$
h=-3\left(a a^{\prime}\right)^{4} a_{x}^{\prime} a_{x}^{\prime \prime 3} \cdot\left(a^{\prime} a^{\prime \prime}\right)\left(a a^{\prime \prime}\right)^{2}
$$

F adoperando la solita equazione identica:

$$
\left(a a^{\prime}\right) a_{x}^{\prime \prime}+\left(a^{\prime} a^{\prime \prime}\right) a_{n}+\left(a^{\prime \prime} a\right) a_{x}^{\prime}=0
$$

troviamo:

$$
h=3\left(a a^{\prime}\right)^{3} a_{x}^{\prime} a_{x}^{\prime \prime}{ }^{2}\left(a^{\prime} a^{\prime \prime}\right)\left(a a^{\prime \prime}\right)^{2}\left\{\left(a^{\prime} a^{\prime \prime}\right) a_{x}-\left(a a^{\prime \prime}\right) a_{x}^{\prime}\right\}
$$

Delle due parti, nelle quali si decompone questa espressione, la prima muta di segno se si fa lo scambio (che evidentemente $\dot{e}$ indifferente) delle a colle $a^{\prime}$; e la seconda muta di segno per lo scambio delle $a^{\prime}$ colle $a^{\prime \prime}$. Dunque ciascuna delle due parti, epperò anche $h$ è identicamente zero; ciò che si roleva provare.

Un altro covariante di quarto grado risulta dallo stesso $k$, ed è il suo determinante Hessiano. Indichiamolo con $\Delta$, ponendo: 


$$
\Delta=2\left(k_{11} k_{22}-k_{1 \%}^{*}\right)
$$

ossia : facendo simbolicamente $k=k_{x}^{i}=h_{x}^{i} \cdots$ :

$$
\Delta=k_{x}^{2} k_{x}^{\prime 2}\left(k k^{\prime}\right)^{2}
$$

Da $k$ ed $u$ scaturisce una serie di covarianti di secondo grado. Da prima, combinando $k$ con $u$, si ottiene la forma quadratica:

$$
\begin{gathered}
l=u_{1111} k_{2222}-4 u_{1112} k_{12: 2}+6 u_{1122} k_{1122}-4 u_{1222} k_{1112}+u_{2222} k_{1111} \\
=a_{x}^{2}(a k)^{4}=l_{2}^{2},
\end{gathered}
$$

e poi combinando $l$ con $k$, ecc.

$$
\begin{aligned}
& m=k_{11} l_{22}-2 k_{12} l_{12}+k_{22} l_{11}=k_{x}^{2}(k l)^{2}=m_{x}^{2}, \\
& n=k_{11} m_{22}-2 k_{12} m_{12}+k_{22} m_{11}=k_{x}^{2}(k m l)^{2}=n_{x}^{2},
\end{aligned}
$$

Ponendo :

$$
\begin{aligned}
& A_{l l}=l_{11} l_{22}-l_{12}^{*}=\frac{1}{2}\left(l l^{\prime}\right)^{2}, \\
& A_{m n}=i n_{11} m n_{22}-m n_{12}^{2}={ }_{4}^{1}\left(m m n^{\prime}\right)^{2} \text {, } \\
& A_{n n}=n_{11} n_{22}-n_{i 2}^{2}=\frac{1}{2}\left(n n^{\prime}\right)^{\prime \prime}, \\
& A_{m n}=\frac{1}{2}\left(m n_{11} n_{22}-2 m_{12} n_{12}+m_{22} n_{11}\right)=\frac{1}{3}(m n)^{2}, \\
& A_{n l}=\frac{1}{2}\left(n_{11} l_{22}-2 n_{22} l_{12}+n_{22} l_{11}\right)=\frac{1}{2}(n l)^{2}, \\
& A_{l n}=\frac{1}{2}\left(l_{11} m_{22}-2 l_{12} m_{12}+l_{22} m_{11}\right)=\frac{1}{2}(l m)^{2},
\end{aligned}
$$

sono questi sei invarianti di $u$, rispettivamente degli ordini $6,10,14,12$, 10,8 . Fra le tre funzioni $l, m, n$ ha luogo una relazione facile a scriversi, i cui coefficienti sono questi medesimi invarianti. Eseguendo il prodotto: 


$$
0=\left|\begin{array}{llll}
l_{11} & l_{12} & l_{22} & 0 \\
m_{11} & n_{12} & n_{22} & 0 \\
n_{11} & n_{12} & n_{22} & 0 \\
x_{2}^{2} & -x_{1} x_{2} & x_{1}^{2} & 0
\end{array}\right|\left|\begin{array}{llll}
l_{22} & -2 l_{12} & l_{11} & 0 \\
m_{22} & -2 n_{12} & 3 n_{11} & 0 \\
n_{22} & -2 n_{12} & n_{11} & 0 \\
x_{1}^{2} & 2 x_{1} x_{2} & x_{2}^{2} & 0
\end{array}\right|,
$$

e dividendo per 4 , si ottiene quella relazione nella forma:

$$
\begin{gathered}
0=\left|\begin{array}{llll}
A_{l l} & A_{l n} & A_{l: l} & l \\
A_{n l} & A_{n i n} & A_{m n} & n \\
A_{n l} & A_{n m} & A_{n n} & n \\
l & m & n & 0
\end{array}\right|=-\Phi, \\
=-\left\{B_{l l} l^{2}+B_{m m} n^{2}+B_{n n} n^{2}+2 B_{m n} m n+2 B_{n z} n l+2 B_{l m} l m\right\},
\end{gathered}
$$

dove le $B$ indicano $i$ determinanti minori formati colle $A$.

Se invece formiamo l'altro prodotto:

$$
\left|\begin{array}{cccc}
l_{11} & l_{12} & l_{22} & 0 \\
n_{11} & n_{12} & n_{22} & 0 \\
n_{11} & n_{12} & n_{22} & 0 \\
x_{1}^{2} & -x_{1} x_{2} & x_{2}^{3} & 1
\end{array}\right|\left|\begin{array}{cccc}
l_{22} & -2 l_{12} & l_{11} & \% \\
m_{22} & -2 m_{12} & m_{11} & \mu \\
n_{22} & -2 n_{12} & n_{11} & \nu \\
x_{1}^{2} & 2 x_{1} x_{2} & x_{2}^{2} & 0
\end{array}\right|
$$

otteniamo:

$$
\left|\begin{array}{llll}
2 A_{l l} & 2 A_{l m} & 2 A_{\eta n} & l \\
2 A_{m l} & 2 A_{m n} & 2 A_{m n} & m \\
2 A_{n l} & 2 A_{n n} & 2 A_{n n} & n \\
l+\lambda & n+\mu & n+\nu & 0
\end{array}\right|=-2\left\{\% \Phi^{\prime}(l)+\mu \Phi^{\prime}(n)+\nu \Phi^{\prime}(n)\right\} .
$$

Ora il primo fattore dell'antecedente prodotto è l'invariante di $15^{\circ}$ ordine (quello d'ordine dispari più piccolo che esista):

$$
2 R=\left|\begin{array}{lll}
l_{11} & l_{12} & l_{22} \\
m_{11} & m_{12} & m_{22} \\
n_{11} & n_{12} & n_{22}
\end{array}\right|
$$


mentre l'altro fattore è il determinante :

$$
-2\left|\begin{array}{ccc}
\lambda & l_{1} & l_{2} \\
\mu & m_{1} & m_{2} \\
\nu & n_{1} & n_{2}
\end{array}\right|
$$

Perció confrontando si ottengono i determinanti funzionali delle $l, m, n$, espressi per mezzo di queste medesime funzioni:

$$
\begin{aligned}
& R\left(m_{1} n_{2}-n_{1} m_{2}\right)=\frac{1}{2} \Phi^{\prime}(l)=B_{l l} l+B_{l m} m+B_{l n} n, \\
& R\left(n_{1} l_{2}-l_{1} n_{2}\right)=\frac{1}{2} \Phi^{\prime}(m)=B_{m l} l+B_{m m} m+B_{m n} n, \\
& R\left(l_{1} m_{2}-m_{1} l_{2}\right)=\frac{1}{2} \Phi^{\prime}(n)=B_{n 2} l+B_{n n} m+B_{n n} n .
\end{aligned}
$$

E pel quadrato di $R$ si trova:

$$
R^{2}=\frac{1}{8}\left|\begin{array}{lll}
l_{11} & l_{12} & l_{22} \\
m_{11} & m_{12} & m_{22} \\
n_{11} & n_{12} & n_{22}
\end{array}\right|\left|\begin{array}{ccc}
l_{22} & -2 l_{12} & l_{11} \\
n_{22} & -2 m_{12} & n_{11} \\
n_{22} & -2 n_{12} & n_{11}
\end{array}\right|=\left|\begin{array}{ccc}
A_{l l} & A_{l m} & A_{l n} \\
A_{m l} & A_{m m} & A_{m n} \\
A_{n l} & A_{m n} & A_{n n}
\end{array}\right| .
$$

$\S 9$,

\section{Relazioni fra gli invarianti.}

Fra i sei invarianti $A_{l l} \ldots$ sono eguali quei due che sono dello stesso ordine, cioè :

$$
A_{n l}=A_{m m}
$$

In fatti è simbolicamente :

$$
A_{n l}=\frac{1}{2}(n l)^{2}=\frac{1}{2}(k m)^{2}(k l)^{2}=\frac{1}{2}\left(m m^{\prime}\right)^{2}=A_{m m} \text {. }
$$

Inoltre $A_{m n}$ ed $A_{n n}$ si possono ridurre a quegli invarianti d'ordine inferiore che si derivano senz' altro dalla funzione $k$, cioè :

$$
\begin{aligned}
& B=\alpha \varepsilon-4 \beta \delta+3 \gamma^{2}=\frac{1}{2}\left(k k^{\prime}\right)^{4}, \\
& C=\left|\begin{array}{l}
\alpha \beta \gamma \\
\beta \gamma \delta \\
\gamma \delta \varepsilon
\end{array}\right|=\frac{1}{6}\left(k k^{\prime}\right)^{2}\left(k^{\prime} k^{\prime \prime}\right)^{2}\left(k^{\prime \prime} k\right)^{2} .
\end{aligned}
$$


A tale uopo rappresentiamo in due modi diversi il covariante quadratico che segue dopo $l, m, n$ :

$$
p=k_{11} n_{22}-2 k_{12} n_{12}+k_{22,2} n_{11} .
$$

Dall'espressione del determinante fumzionale $l_{1} m_{2}-m_{1} l_{2}$ per mezzo di $l, m, n$, segue l'equazione:

$$
B_{n l} m+B_{n m} n+B_{n n} p=R\left\{k_{11}\left(l_{1} m_{2}-m_{1} l_{2}\right)_{22} \ldots\right\} .
$$

L'espressione simbolica della quantit‘் fra parentesi è :

$$
k_{x}^{2}(l m)(k l)(k m)
$$

e per mezzo della relazione identica:

$$
k_{x}(m)=l_{x}(k m)-m_{x}(k l),
$$

si cambia in :

$$
\begin{gathered}
k_{x}(k l)(k m)\left\{l_{x}(k m)-m_{x}(k l)\right\} \\
=n_{x} l_{x}(n l)-m_{x} m_{x}^{\prime}\left(m^{\prime} m\right)=n_{1} l_{2}-n_{2} l_{1} .
\end{gathered}
$$

Introducendo per quest'ultimo determinante il suo valore, si ha l'equazione :

$$
B_{n l} i n+B_{n m} n+B_{i n} p=B_{m l} l+B_{m i n} u+B_{m u t} n
$$

ossia :

$$
B_{u n} p=B_{m l} l+\left(B_{m u}-B_{n l}\right) m .
$$

Quest'equazione è degna d'attenzione sotto più aspetti. Nell' espressione di $p$ non entra più $n$. Siccome poi nel ricavare questa formola non si è fatto uso della derivazione di $l, m, \ldots$ da $k$ ed $u$, ma piuttosto della circostanza che $l, m, n, p$ sono consecutivi nella serie dei covarianti di secondo grado, cosi si fa chiaro il teorema:

Nella serie dei covarianti di secondo grado $l, m, n, p \ldots$ ciascuno è composto linearmente coi due che precedono di due e di tre posti.

La seconda espressione di $p$ scaturisce da due formole che adopreremo anche in seguito e che, spettando alla teoria delle forme di quarto grado, 
qui saranno solamente citate. Se $k$ è una qualsivoglia forma di quarto grado, e :

$$
\Delta=2\left(k_{11} k_{22}-k_{12}^{2}\right)
$$

se $B$ e $C$ sono gli invarianti di $k$ già menzionati; e inoltre se $\phi$ è una funzione di secondo grado, e:

$$
\psi=k_{11} \hat{\phi}_{22}-2 k_{12} \phi_{12}+k_{22} \phi_{11} .
$$

si avranno le due equazioni :

$$
\begin{aligned}
& \Delta_{11} \phi_{22}-2 \Delta_{12} \dot{\phi}_{12}+\Delta_{22} \dot{\phi}_{11}=k_{11} \psi_{22}-2 k_{12} \psi_{12}+k_{22} \psi_{11}-\frac{2}{3} B \phi \\
& \Delta_{11} \psi_{22}-2 \Delta_{12} \psi_{12}+\Delta_{22} \psi_{11}={ }_{3}^{1} B \psi+2 G \phi .
\end{aligned}
$$

Ponendo qui $\phi=l, \psi=m$, ovvero $\phi=m, \psi=n$, si ottengono le quattro equazioni:

$$
\begin{aligned}
\Delta_{11} l_{22}-2 \Delta_{12} l_{12}+\Delta_{22} l_{11} & =n-\frac{2}{3} B l \\
\Delta_{11} m_{22}-2 \Delta_{12} m_{12}+\Delta_{22} m_{11} & =\frac{1}{3} B m+2 G l \\
& =p-\frac{2}{3} B m \\
\Delta_{11} n_{22}-2 \Delta_{12} n_{12}+\Delta_{22} n_{11} & =\frac{1}{3} B n+2 G m .
\end{aligned}
$$

Dunque è :

$$
p=B m+2 C l
$$

e confrontando questa colla precedente espressione di $p$, si trova:

$$
\begin{aligned}
& B_{m l}=2 C \cdot B_{n n}, \\
& B_{m n}-B_{n l}=B . B_{n n} .
\end{aligned}
$$

Pongansi questi valori nelle equazioni identiche :

$$
\begin{aligned}
& A_{l l} B_{m l}+A_{m l} B_{n ? m}+A_{n l} B_{m n}=0, \\
& A_{m l} B_{m l}+A_{m n} B_{m m}=A_{n l} B_{n l}+A_{m n} B_{n n},
\end{aligned}
$$

e si osservi che $A_{n z}=A_{m m}$; si avranno le due equazioni :

$$
\begin{aligned}
& A_{n m}=B A_{I n n}+2 C A_{l l}, \\
& A_{n n}=B A_{m m}+2 C A_{m l},
\end{aligned}
$$

mediante le quali $A_{n m}$ ed $A_{n n}$ sono ricondotti ad $A_{l l}, A_{m l}, A_{m m}$ ed a $B, C$. 
Siccome la funzione $u$ non pud possedere che quattro invarianti indipendenti fra loro, cosi fra i cinque invarianti suddetti dovrà verificarsi un' altra relazione. In ciò che precede, non essendo noi risaliti al fatto che $l, k$ traggono origine da $u$, le forme $A_{l l}, A_{m l}, A_{m n}, B, C$ sono gli invarianti simultanei di $k, l$, indipendenti fra loro. Perció l'ultima relazione deve necessariamente tener calcolo di tale origine. Se ammettiamo che, per $u$, oltre agli invarianti degli ordini $4(B), 6\left(C, A_{n}\right), 8\left(A_{l_{m}}\right), 10\left(A_{m n n}\right)$, ne esista anche uno di second' ordine :

$$
A=\frac{1}{2}\left(a a^{\prime}\right)^{6}=a g-6 b f+15 c e-10 d^{2},
$$

allora il problema che dobbiamo risolvere consiste nello scoprire due relazioni fra $A, B, C, A_{l l}, A_{\text {mm }}, A_{m m}$. Questo problema si risolve nella seguente maniera.

Secondo il processo più volte impiegato, si ha:

$$
\begin{gathered}
\left.\left.A k=\frac{1}{2}\left(a a^{\prime}\right)^{6} \cdot k_{x}^{4}=\frac{1}{2}\left(a a^{\prime}\right)^{2}\right\}(a k) a_{x}^{\prime}-\left(a^{\prime} k\right) a_{x}\right\}^{4} \\
=\left\{(a k)^{4} a_{x}^{\prime 4}-4(a k)^{3}\left(a^{\prime} k\right) a_{x} a_{x}^{\prime 3}+3(a k)^{2}\left(a^{\prime} k\right) a_{x}^{2} a_{x}^{\prime 2}\right\}\left(a a^{\prime}\right)^{2} .
\end{gathered}
$$

Siccome poi dalla:

$$
l_{i}=3\left(a a^{\prime}\right)^{2} a_{x}^{2} \alpha^{\prime 2}
$$

si ricava mediante la differenziazione:

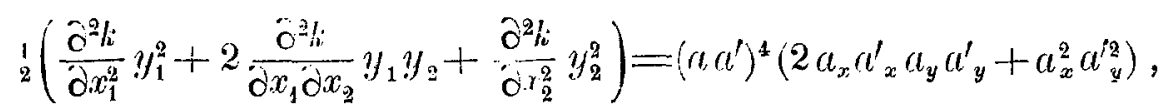

cosi, ponendo $k_{x} k_{2},-k_{x} k_{1}$ invece di $y_{1}, y_{2}$ si trova:

$$
\begin{aligned}
& \Delta=\left(a a^{\prime}\right)^{4} k_{x}^{2}\left(2(a k)\left(a^{\prime} k\right) a_{x} a_{x}^{\prime}+\left(a^{\prime} k\right)^{2} a_{x}^{\prime}\right) \\
& =\left(a a^{\prime}\right)^{2}\left(2(a k)\left(a^{\prime} k\right) a_{x} a_{x}^{\prime}+\left(a^{\prime} k\right)^{2} a_{x}^{2}\right)\left((a k)^{2} a_{x}^{\prime 2}-2(a k)\left(a^{\prime} k\right) a_{x} a_{x}^{\prime}+\left(a^{\prime} k\right)^{2} a_{x}^{2}\right) \\
& =\left(a a^{\prime}\right)^{2}\left(a^{\prime} k\right)^{4} a_{x}^{\prime}+2\left(a a^{\prime}\right)^{2}\left(a^{\prime} k\right)^{3}(a k) a_{x}^{2} a_{x}^{\prime}-3\left(a a^{\prime}\right)^{2}\left(a^{\prime} k\right)^{2}(a k)^{2} a_{x}^{2} a_{x}^{\prime 2} .
\end{aligned}
$$

Da ultimo ricaviamo dal corariante identicamente nullo $h$ la forma parimente nulla $(h a)^{3} h_{x} a_{x}^{3}$, ossia l'equazione identica:

$$
0=(a k)^{3}\left(a a^{\prime}\right)^{3} k_{x} a_{x}^{\prime 3}+3(a k)^{3}\left(a a^{\prime}\right)^{2}\left(k a^{\prime}\right) a_{x} a_{x}^{\prime 3}
$$


la quale, in virtù della:

$$
\left(a a^{\prime}\right) k_{x}=(a k) a_{x}^{\prime}-\left(a^{\prime} k\right) a_{x},
$$

si muta in :

$$
0=(a k)^{4}\left(a a^{\prime}\right)^{2} a_{x}^{\prime 4}-4\left(a a^{\prime}\right)^{2}(a k)^{3}\left(a^{\prime} k\right) a_{x} a_{x}^{\prime 3} .
$$

Ora, se per brevità si pone:

$$
\begin{gathered}
\rho=\left(a a^{\prime}\right)^{2}(a k)^{4} a_{x}^{\prime 4}, \quad \sigma=(a k)^{3}\left(a^{\prime} k\right)\left(a a^{\prime}\right)^{2} a_{x} a_{x}^{\prime 3}, \\
\tau=\left(a a^{\prime}\right)^{2}(a k)^{2}\left(a^{\prime} k\right)^{2} a_{x}^{2} a_{x}^{\prime 2},
\end{gathered}
$$

per le cose precedenti avremo le equazioni:

$$
\begin{gathered}
A k=\rho-4 \sigma+3 \tau, \quad 0=\rho-4 \sigma, \\
\Delta=\rho+2 \sigma-3 \tau,
\end{gathered}
$$

alle quali si puod aggiungere la definizione:

$$
u_{11} l_{22}-2 u_{12} l_{12}+u_{22} l_{11}=\rho .
$$

Si ha dunque :

$$
\tau=\frac{1}{3} A k, \quad \rho=\frac{2}{3}(\Delta+A k),
$$

e quindi la relazione fra covarianti :

$$
u_{11} l_{22}-2 u_{12} l_{12}+u_{22} l_{11}=\frac{2}{3}(\Delta+A k) .
$$

Se si scrive questa equazione nella forma simbolica:

$$
a_{x}^{4}(a l)^{2}=\frac{2}{3}\left(k k^{\prime}\right)^{2} k_{x}^{2} k_{x}^{\prime 2}+\frac{2}{3} A k_{x}^{4},
$$

sostituendo $k_{2},-k_{1}$ in luogo di $x_{1}, x_{2}$, si otterrà :

$$
(a k)^{4}(a l)^{2}=\frac{2}{3}\left(k k^{\prime}\right)^{2}\left(k k^{\prime \prime}\right)^{2}\left(k^{\prime} k^{\prime \prime}\right)^{2}+\frac{2}{3} A\left(k k^{\prime}\right)^{4},
$$

ossia :

$$
A_{n}=2\left(C+\frac{A B}{3}\right)
$$


che è una delle relazioni cercate. L' altra si ricava dalla considerazione dell'espressione:

$$
\begin{aligned}
& u_{11} m_{22}-2 u_{12} m_{12}+u_{22} m_{11}=a_{x}^{4}(a m)^{2}=a_{x}^{4}(a k)^{2}(k l)^{2} \\
& =(a k)^{2} a_{x}^{2}\left\{k_{x}(a l)-l_{x}(a k)\right\}^{2} \\
& =(a k)^{2}(a l)^{2} a_{x}^{2} k_{x}^{2}+(a k)^{4} a_{x}^{2} l_{x}^{2}-2(a k)^{3}(a l) a_{x}^{2} k_{x} l_{x} .
\end{aligned}
$$

La seconda parte di quest'espressione è a dirittura:

$$
(a k)^{4} a_{x}^{2} l_{x}^{2}=l_{x}^{\prime 2} l_{x}^{2}=l^{2}
$$

La prima nasce dalla forma:

$$
\phi=u_{11} l_{22}-2 u_{12} l_{12}+u_{22} l_{11}=\alpha_{x}^{4}(a l)^{2},
$$

mediante l'operazione :

$$
\phi_{11} k_{22}-2 \phi_{12} k_{12}+\phi_{22} k_{11}
$$

epperd è eguale a :

$$
\frac{2}{3}\left(\Delta_{11} k_{22}-2 \Delta_{12} k_{12}+\Delta_{22} k_{11}\right)+\frac{2}{3} A \Delta
$$

ossia eguale a :

$$
\frac{2}{9} B l i+\frac{\circ}{3} A \Delta
$$

perchè, in virtù della teoria delle forme di quarto grado, l'espressione fra parentesi ha il valore $\frac{1}{3} B l$.

La terza parte si ottiene col sussidio del covariante $h=(a l k)^{3} a_{x}^{3} k_{x}$, che è identicamente nullo. Da questo si trae subito:

$$
\begin{gathered}
0=4\left(h_{1} l_{2}-l_{1} h_{2}\right)=(a k)^{3} a_{x}^{2}\left\{3(a l) k_{x} l_{x}+(k l) a_{x} l_{x}\right\} \\
=(a k)^{3} a_{x}^{2}\left\{4(a l) k_{x} l_{x}-(a l k) l_{x}^{2}\right\}
\end{gathered}
$$

dunque :

$$
(a k)^{3}(a l) \alpha_{x}^{2} k_{x} l_{x}=\frac{1}{4}(a k)^{4} \alpha_{x}^{2} l_{x}^{2}={ }_{4}^{1} l^{2}
$$


Laonde si ha finalmente la formola:

$$
i_{12} m n_{22}-2 u_{12} m_{12}+u_{22} m_{11}=\frac{1}{2} l^{2}+\frac{9}{9} B k+\frac{\pi}{3} A \Delta
$$

la quale simbolicamente equivale a :

$$
(a m)^{2} a_{x}^{4}=\frac{1}{2} l_{x}^{2} l_{x}^{\prime 2}+\frac{9}{9} B k_{x}^{4}+\frac{2}{3} A\left(k k^{\prime}\right)^{2} k_{x}^{2} k_{x}^{\prime 2}
$$

dove, ponendo di nuovo $k_{2},-k_{1}$ invece di $x_{1}, x_{2}$, risulta la relazione fra invarianti :

$$
(l m)^{2}=\frac{1}{2}(l m)^{2}+\frac{2}{3} B\left(k k^{\prime}\right)^{4}+\frac{2}{3} A\left(k k^{\prime}\right)^{2}\left(k k^{\prime \prime}\right)^{2}\left(k^{\prime} k^{\prime \prime}\right)^{2},
$$

ossia :

$$
A_{\text {lum }}={ }_{9}^{4} B^{2}+4 A G \text {. }
$$

In conclusione vediamo essere conveniente di assumere per fondamentali gli invarianti :

$$
\begin{gathered}
A\left(\text { di } 2^{\circ} \text { ordine }\right), \quad B\left(\text { di } 4^{\circ} \text { ordine }\right), \quad C\left(\text { di } 6^{\circ} \text { ordine }\right), \\
D=A_{m m}\left(\text { del } 10^{\circ} \text { ordine }\right)
\end{gathered}
$$

come i più semplici, mentre l'invariante $A_{m l}$ di $8^{\circ}$ ordine si esprime razionalmente per mezzo dei precedenti.

Quindi le espressioni finali degli invarianti divengono:

$$
\begin{aligned}
& A_{l l}=2 C+\frac{9}{3} A B, \quad A_{m l}=\frac{4}{9} B^{2}+4 A C, \quad A_{n l}=A_{n m b}=D, \\
& A_{n n l}=4\left(C^{2}+\frac{4}{3} A B C+{ }_{9}^{1} B^{3}\right), \quad A_{m n}=B D+{ }_{9}^{8} B^{2} C+8 A C^{2} .
\end{aligned}
$$

E da ultimo:

$$
R^{2}=\left|\begin{array}{lll}
2 C+\frac{2}{3} A B & \frac{4}{9} B^{2}+4 A C & D \\
\frac{4}{9} B^{2}+4 A C & D & 4 C^{2}+\frac{16}{3} A B C+\frac{4}{9} B^{3} \\
D & 4 C^{2}+\frac{16}{3} A B C+\frac{4}{9} B^{3} & B D+\frac{8}{9} B^{2} C+8 A C^{2}
\end{array}\right| .
$$


$\$ 10$.

Rappresentazioni tiplche.

Se per un covariante $\phi$ si conoscono le forme:

$$
\begin{aligned}
& \phi_{l}=\phi_{11} l_{22}-2 \phi_{12} l_{12}+\phi_{22} l_{11}, \\
& \phi_{i l}=\phi_{11} m_{22}-2 \phi_{12} m_{12}+\phi_{22} m_{11}, \\
& \phi_{n}=\phi_{11} n_{22}-2 \phi_{12} n_{12}+\phi_{22} n_{11},
\end{aligned}
$$

nella loro rappresentazione tipica per mezzo di $l, m, n$, si puó facilmente assegnare anche l'espressione di $\phi$. In fatti, se alle equazioni precedenti si aggiunge la :

$$
\phi=\phi_{11} x_{1}^{2}+2 \phi_{12} x_{1} x_{2}+\phi_{22} x_{2}^{2},
$$

eliminando $\phi_{11}, \phi_{12}, \phi_{22}$, risulta:

$$
0=\left|\begin{array}{llll}
l_{22} & -l_{12} & l_{11} & \phi_{l} \\
m_{22} & -m_{12} & m_{11} & \phi_{m b} \\
n_{22} & -n_{12} & n_{11} & \phi_{n} \\
x_{1}^{2} & x_{1} x_{2} & x_{2}^{2} & \phi
\end{array}\right|,
$$

ovvero, confrontando colla fine del $\S 6$, e moltiplicando per $R$ :

$$
R^{2} \cdot \phi=-\left|\begin{array}{llll}
A_{l l} & A_{i_{m}} & A_{i n} & \phi_{l} \\
A_{m l} & A_{m m} & A_{m n} & \phi_{n} \\
A_{n l} & A_{n m} & A_{n n} & \phi_{n} \\
l & m & n & 0
\end{array}\right| .
$$

Indichiamo per un momento con ciascuno de' simboli $\mu, v, .$. uno qualunque degli indici $l, m, n$, e con ciascuno de' simboli $l_{\mu}, l_{\nu}, \ldots$ una qualunque delle funzioni $l, m, n$; allora potremo scrivere l'equazione precedente anche cosi:

$$
R^{2} \cdot \phi=\mathbf{\Sigma}_{\mu} \underset{\nu}{\mathbf{\Sigma}} B_{\mu \nu} l_{\mu} \phi_{\nu} .
$$

Se analogamente $\phi_{l}, \phi_{n l}, \phi_{n}$ sono date per mezzo di $\phi_{l l}, \phi_{l n}=\phi_{m l}$, ecc., sarà anche: 


$$
R^{2} \cdot \phi_{\nu}=\sum_{\varrho} \underset{\sigma}{\mathbf{g}} B_{\rho \sigma} l_{\rho} \phi_{\nu \sigma}
$$

epperò :

$$
\begin{aligned}
& R^{4} \cdot \phi=\sum_{\mu} \sum_{\nu} \sum_{\varrho} \underset{\sigma}{\mathbf{y}} B_{\mu \nu} B_{\varphi \sigma} l_{\mu} l_{\varphi} \phi_{\nu \sigma} \\
& =\sum_{\mu \nu \rho \sigma} B_{\mu \rho} B_{\sigma \nu} l_{\mu} l_{\rho} \phi_{\sigma \nu}-R^{2} \sum_{\mu \nu \rho \sigma}^{\mathbf{\Sigma}} B_{\mu \rho}, \sigma \nu l_{\mu} l_{\rho} \phi_{\nu \sigma} .
\end{aligned}
$$

Ora, siccome $\sum \sum B_{\mu \rho} l_{\mu} l_{\varphi}$ è identicamente zero, così l'equazione si puó dividere per $R^{2}$, onde si ha:

$$
R^{2} \cdot \bar{\phi}=-\sum_{\mu \nu \rho \sigma} B_{\mu \rho ; \sigma \nu} l_{\mu} l_{\varphi} \phi_{\nu \sigma}
$$

Quindi, scrivendo per un momento $\overline{\phi_{\nu} \cdot \phi_{\sigma}}$ simbolicamente in luogo di $\phi_{\nu \sigma}$, questa formola diviene :

$$
R^{2} \cdot \phi=-\left|\begin{array}{lllll}
A_{l l} & A_{l m l} & A_{l n} & l & \overline{\phi_{l}} \\
A_{m l} & A_{m m} & A_{m n} & m & \overline{\phi_{m l}} \\
A_{n l} & A_{n m} & A_{n n} & n & \overline{\phi_{n}} \\
l & m & n & 0 & 0 \\
\overline{\phi_{l}} & \frac{m}{\bar{\phi}_{m}} & \frac{\bar{\phi}_{n}}{n} & 0 & 0
\end{array}\right|
$$

Queste formole bastano per la rappresentazione di $k, \Delta$ ed $u$. In fatti si ha gid per $k$ :

epperò :

$$
\begin{aligned}
& k_{11} l_{22}-2 k_{12} l_{12}+k_{22} l_{11}=m, \\
& k_{11} m_{22}-2 k_{12} m_{12}+k_{22} m_{11}=n, \\
& k_{11} n_{22}-2 k_{12} n_{12}+k_{22} n_{11}=p=B r l+2 C l ;
\end{aligned}
$$

$$
R^{2} \cdot k=-\left|\begin{array}{llll}
A_{l l} & A_{l m} & A_{l n} & m \\
A_{m l} & A_{m n} & A_{m n} & n \\
A_{n l} & A_{2 n} & A_{n n} & B m+2 C l \\
l & m & n & 0
\end{array}\right|
$$

Poi si aveva:

$$
\begin{aligned}
& \Delta_{11} l_{22}-2 \Delta_{12} l_{12}+\Delta_{22} l_{11}=n-\frac{2}{3} B l, \\
& \Delta_{11} m_{22}-2 \Delta_{12} m_{12}+\Delta_{22} m_{11}=\frac{1}{3} B m+2 C l, \\
& \Delta_{11} n_{22}-2 \Delta_{12} n_{12}+\Delta_{22} n_{11}=\frac{1}{3} B n+2 C m,
\end{aligned}
$$


dunque :

$$
R^{2} \cdot \Delta=-\left|\begin{array}{cccc}
A_{l l} & A_{l m} & A_{l n} & n-\frac{2}{3} B l \\
A_{m l} & A_{m n l} & A_{m n} & \frac{1}{3} B m+2 C l \\
A_{n l} & A_{n n} & A_{n n} & \frac{1}{3} B n+2 C m \\
l & i n & n & 0
\end{array}\right|
$$

Finalmente è :

$$
\begin{aligned}
& u_{l}=u_{11} l_{22}-2 u_{12} l_{12}+u_{22} l_{11}=\frac{2}{3} \Delta+\frac{2}{3} A k, \\
& u_{m}=u_{11} m_{22}-2 u_{12} m_{12}+u_{22} m_{11}=\frac{1}{2} l^{2}+\frac{2}{9} B k+\frac{2}{3} A \Delta .
\end{aligned}
$$

A queste equazioni si deve anzitutto aggiungere il valore di $u_{n}$. Seguends, precisamente la stessa via adottata per $u_{a}(\$ 9)$, troviamo:

$$
u_{1 k}=u_{11} n_{22}-2 u_{12} n_{12}+u_{22} n_{11}=m l+\frac{2}{9} B \Delta-\frac{2}{3} C k \text {. }
$$

Ora combinando di nuovo $u_{l}, u_{n k}, u_{n}$ con $l, m, n$, si trovano espressioni nelle quali non restano incognite che quelle parti che nascono dai termini $l^{2}, m l$. Ma, se $\phi, \psi, \chi$ sono tre funzioni qualunque di secondo grado, si ha:

$$
\begin{gathered}
\frac{1}{2}\left(\frac{\partial^{2} \varphi \cdot \psi}{\partial x_{1}^{2}} \chi_{22}-2 \frac{\partial^{2} \varphi \cdot \psi}{\partial x_{1} \partial x_{2}} \chi_{12}+\frac{\partial^{2} \cdot \dot{\psi}}{\partial x_{2}^{2}} \chi_{11}\right) \\
=3 \psi\left(\phi_{11} \chi_{22}-2 \phi_{12} \chi_{12}+\phi_{22} \chi_{11}\right)+3 \phi\left(\psi_{11} \chi_{22}-2 \psi_{12} \chi_{12}+\psi_{22} \chi_{11}\right)- \\
-2 \chi\left(\phi_{11} \psi_{22}-2 \phi_{12} \psi_{12}+\phi_{22} \psi_{11}\right)
\end{gathered}
$$

com'e facile a provarsi. Quindi sarà :

$$
\begin{array}{ll}
(l l)_{l}=\frac{1}{3} A_{n l} \cdot l, & (m l)_{l}=\frac{1}{3} A_{m l} \cdot l+A_{l l} \cdot m, \\
(l l)_{m}=2 A_{l m} \cdot l-\frac{2}{3} A_{l l} \cdot m, & (m l)_{m b}=A_{m m} \cdot l+\frac{1}{3} A_{m l} \cdot m, \\
(l l)_{n}=2 A_{l n} \cdot l-\frac{2}{3} A_{l l} \cdot n, & (m l)_{n}=A_{m n} \cdot l+A_{n l} \cdot m-\frac{2}{3} A_{m l} \cdot n,
\end{array}
$$

e per conseguenza: 


$$
\begin{aligned}
& u_{l l}=-\frac{4}{9} B l+-\frac{9}{3} A m+\frac{2}{3} n, \\
& u_{m l}=\frac{4}{3} G l+\frac{2}{9} B m+\frac{2}{3} A n, \\
& u_{n l}=\frac{4}{3} A C l+\left(\frac{2}{3} A B+\frac{4}{3} C\right) m+\frac{2}{9} B n, \\
& u_{m m}=\left(\frac{4}{9} B^{2}+\frac{16}{3} A C\right) l-\frac{2}{3} C m+\frac{2}{9} B n, \\
& u_{m n}=\left(D+\frac{4}{9} B C\right) l+\left(\frac{4}{3} A C+\frac{2}{9} B^{2}\right) m-\frac{2}{3} C n, \\
& u_{n n}=\left(\frac{4}{9} B^{3}+\frac{16}{3} A B C+\frac{8}{3} C^{2}\right) l+\left(D-\frac{2}{9} B C\right) m-\left(\frac{2}{9} B^{2}+\frac{8}{3} A C\right) n .
\end{aligned}
$$

Quindi, introducendo le funzioni di invarianti :

$$
\begin{aligned}
& E=\frac{2}{3} D-\frac{16}{3} A^{2} C-{ }_{9}^{4} A B^{2}+\frac{4}{9} B C, \\
& F=\frac{2}{3} A D-\frac{90}{9} A B C-\frac{4}{3} C^{2}-\frac{16}{81} B^{3},
\end{aligned}
$$

risulta per $u$ la forma tipica :

$$
\begin{aligned}
R^{2} \cdot u= & E\left\{-8 C^{2} l^{3}-C m^{3}+n^{3}+\left({ }_{2}^{9} D-y B C\right) l^{2} m-2 B^{2} l m^{2}-2 B l n^{2}+B m^{2} n-6 C l m n\right\} \\
& +3 F\left\{\left(B^{2}+12 A C\right) l^{2} m-4 C l^{2} n-4 C l m^{2}+m n^{2}-2 B l m n\right\},
\end{aligned}
$$

mentre la relazione fra $l, m, n$ si muta nella:

$0=E\left\{\left(\frac{3}{2} B D+\frac{1}{3} B^{2} C\right) l^{2}+\left(4 B C-\frac{3}{2} D\right) m^{2}+3 C n^{2}+\frac{5}{3} B^{2} m n+(2 B C-3 D) n l+12 C^{2} l m\right\}$ $+F\left\{\left(B^{3}+12 A B C+12 C^{2}\right) l^{2}-12 A C m^{2}+B n^{2}+12 C m n-2\left(B^{2}+12 A C\right) n l+4 B C l m\right\}$.

Nello stesso tempo $R^{2}$, espresso per mezzo di $E, F$, assume la forma semplice :

$$
R^{2}=\left(\frac{15}{2} B C-\frac{9}{4} D\right) E^{2}-\left(\frac{3}{2} B^{2}+18 A C\right) E F-18 C F^{2}
$$

$\S 11$.

Caso eccezionale: $R=0$. Seconda mappresentazione di $u$.

La precedente rappresentazione tipica di $u$ diventa impossibile se $R$ è nullo, epperò se ha luogo fra $l, m, n$ una relazione lineare. Supponiamo da prima che $m$ ed $l$ non siano nè proporzionali fra loro, nè identicamente nulle. Al- 
lora $n$ è una funzione lineare di $l$ ed $m$, e per conseguenza non può essere adoperata. Invece sí potrà in tal caso usare come terza funzione il determinante :

$$
s=l_{1} m_{2}-m_{1} l_{2}
$$

il quale (cfr. $\$ 1$ ) è legato con $l, m$ mediante l' equazione di secondo grado:

$$
\$^{2}=-\left(A_{l l} m^{2}-2 A_{l_{m}} m l+A_{m m} l^{2}\right)
$$

Cominceremo dall'eseguire in generale questa trasformazione di $u$. In luogo di $R^{2}$ subentra il determinante:

$$
\left|\begin{array}{ccc}
A_{l l} & A_{m l} & A_{\vartheta l} \\
A_{l m} & A_{m m} & A_{\vartheta m} \\
A_{l \vartheta} & A_{m \vartheta} & A_{\vartheta \vartheta}
\end{array}\right|,
$$

nel quale $A_{\imath \vartheta}$, ecc. indicano le espressioni formate analogamene ad $A_{l n}$, ecc. Ma pel $\$ 1$ si ha:

$$
A_{l \vartheta}=0, \quad A_{m \vartheta}=0, \quad A_{\vartheta \vartheta}=A_{l l} A_{m m}-A_{m l}^{2},
$$

onde il valore del determinante superiore è a dirittura $A_{\vartheta_{\vartheta}}^{2}=B_{n n}$. Dunque questa trasformazione è possibile ogni qualvolta $A_{\vartheta \vartheta}$ non sia nullo.

Posto per una funzione qualunque $\phi$ :

$$
\phi_{14}=\phi_{11} \curvearrowright_{22}-2 \phi_{12} \curvearrowright_{12}+\phi_{22} \curvearrowright_{11}
$$

si ha la formola :

$$
A_{9,9}^{2} \cdot \phi=-\left|\begin{array}{llll}
A_{l l} & A_{l n} & 0 & \phi_{l} \\
A_{m l} & A_{m m} & 0 & \phi_{n n} \\
0 & 0 & A_{\vartheta, 9} & \phi_{9} \\
l & m & \curvearrowright & 0
\end{array}\right|
$$

vale a dire che, dopo aver diviso per $A_{\vartheta \vartheta}$, è :

$$
A_{\vartheta \vartheta} \cdot \phi=\$ \phi_{\vartheta}+m\left(A_{l l} \phi_{m}-A_{m !} \phi_{l}\right)-l\left(A_{m l} \phi_{m}-A_{m m} \phi_{l}\right):
$$

e quindi anche: 


$$
\begin{aligned}
A_{\vartheta \vartheta}^{2} \phi & =\$^{2} \phi_{\vartheta \vartheta}+2 \$ m\left(A_{l l} \phi_{m \vartheta}-A_{m l} \phi_{l \vartheta}\right)-2 \$ l\left(A_{m l} \phi_{m \vartheta}-A_{m m} \phi_{l \vartheta}\right) \\
& +\left(A_{l l} \phi_{m m}-2 A_{l m} \phi_{m l}+A_{m n} \phi_{l l}\right)\left(A_{l l} m^{2}-2 A_{m l} m l+A_{m m} l^{2}\right) \\
& -A_{\vartheta \vartheta}\left(m^{2} \phi_{l l}-2 m l \phi_{m l}+l^{2} \phi_{m m}\right) \\
& =2 \$^{2} \phi_{\vartheta \vartheta}-A_{\vartheta \vartheta}\left(m^{2} \phi_{l l}-2 m l \phi_{m l}+l^{2} \phi_{m m}\right) \\
& +2 \$ m\left(A_{l l} \phi_{m \vartheta}-A_{m l} \phi_{l \vartheta}\right)-2 \$ l\left(A_{m l} \phi_{m \vartheta}-A_{m m} \phi_{l \vartheta}\right)
\end{aligned}
$$

essendo identicamente :

$$
\phi_{\vartheta \vartheta}=-\left(A_{n l} \phi_{m m}-2 A_{m l} \phi_{m l}+A_{m m} \phi_{n l}\right) .
$$

Ma, se nella formola:

$$
A_{\vartheta \vartheta} \phi=\$ \phi_{\vartheta}+m\left(A_{l l} \phi_{m}-A_{m l} \phi_{l}\right)-l\left(A_{m l} \phi_{m}-A_{m m} \phi_{l}\right)
$$

poniamo in luogo della funzione arbitraria $\phi$ la funzione $\phi 4$, del pari arbitraria, e moltiplicata per 2 l' equazione risultante la sottraggiamo dall'equazione che dà $A^{2} \vartheta \vartheta \cdot \phi$, otterremo la formola semplice:

$$
A_{\vartheta \rightarrow} \cdot \phi=2 \& \cdot \phi_{9}-\left(m^{2} \phi_{n}-2 \ln \phi_{m l}+l^{2} \phi_{m n n}\right)
$$

quindi anche:

$$
A_{\vartheta \vartheta} \cdot \phi_{\vartheta}=2 \$ \phi_{\vartheta \vartheta}-\left(m^{2} \phi_{l, \vartheta}-2 \ln \phi_{m \vartheta \vartheta}+l^{2} \phi_{m m \vartheta}\right)
$$

Ossia sarà :

$A_{\vartheta \vartheta}^{2} \cdot \phi=4 \$^{2} \phi_{\vartheta \vartheta}-\left(m^{2} \phi_{l l}-2 l m \phi_{l m}+l^{2} \phi_{m m}\right) A_{\vartheta \vartheta}-2 \curvearrowright\left(m^{2} \phi_{l l}-2 m l \phi_{m l}+l^{2} \phi_{m m \vartheta}\right)$.

Applicando questa formola al $u$, si ha:

$$
\begin{aligned}
u_{\vartheta \vartheta}=\frac{2}{3} B E l-F m & -E n \\
m^{2} u_{l l}-2 m l u_{m l}+l^{2} u_{m m} & =\frac{4}{3} A C l^{3}+\frac{2}{3} A m^{3}+\frac{2}{3}(A B-2 G) l^{2} m \\
& -\frac{8}{9} B l m^{2}+\frac{2}{3} n\left(m^{2}-2 A l m+\frac{1}{3} B l^{2}\right), \\
m^{2} u_{l l \vartheta}-2 m l u_{l m}+l^{2} u_{m m} & =\frac{2}{3} R\left(m^{2}-2 A l m+\frac{1}{3} B l^{2}\right) .
\end{aligned}
$$

Quindi la formola per $u$ diviene:

$$
\begin{aligned}
A^{2} \vartheta v & =4 \Omega^{2}\left(\frac{2}{3} B E l-F m-E n\right)-\left(\frac{2}{3} n A \vartheta \vartheta+\frac{1}{3} R S\right)\left(m^{2}-2 A l m+\frac{1}{3} B l^{2}\right)- \\
& -{ }_{3}^{2} A \vartheta \vartheta\left\{2 A C l^{3}+(A B-2 G) l^{2} m-\frac{4}{3} B l m^{2}+A m^{3}\right\} .
\end{aligned}
$$


Se s'introduce anche il valore di $n$ preso dalla formola $(\$ 8)$ :

$$
R S=B_{n l} \cdot l+B_{n m} \cdot m+B_{n n} \cdot n,
$$

si ottiene la seguente rappresentazione di $u$ per mezzo di $l, m, \curvearrowright$ :

$$
\begin{aligned}
& A_{\vartheta \vartheta}^{3} \cdot u=R \&\left\{4 E \$^{2}+\left(2 m^{2}-4 A l m+\frac{2}{3} B l^{2}\right) A_{\vartheta \vartheta}\right\} \\
& +4 \nwarrow^{2}\left\{\left({ }_{3}^{2} B E B_{n n}+E B_{l n}\right) l+\left(-F B_{n n}+E B_{m n}\right) m\right\} \\
& +\frac{2}{3} A_{\vartheta \vartheta}\left(B_{n l} l+B_{n m} m\right)\left(m^{2}-2 l m A+\frac{1}{3} B l^{2}\right) \\
& -\frac{2}{3} A_{4,2}^{2}\left\{2 A C l^{3}+(A B-2 C) l^{2} m-\frac{4}{3} B l m^{2}+A m^{3}\right\} \text {, }
\end{aligned}
$$

che dev'essere combinata coll'equazione:

$$
\$^{2}=-\left(A_{n} m^{2}-2 A_{m m} m l+A_{m m} l^{2}\right) .
$$

Consideriamo ora particolarmente il caso di $R=0$. Ponendo per $S^{2}$ il suo valore, troviamo:

$$
A_{9,9}^{3} \cdot u=P l^{3}+3 Q l^{2} m+3 S l m^{2}+T m^{3},
$$

dove :

$$
\begin{aligned}
& P=-4 A_{m m} E\left({ }_{3}^{2} B B_{n n}+B_{n l}\right)+\frac{2}{9} B B_{n l} B_{n n}-{ }_{3}^{4} A C B_{n n}^{2} \text {, } \\
& 3 Q=8 A_{m l} E\left(\frac{2}{3} B B_{n n}+B_{n l}\right)-4 A_{m m}\left(E B_{m n}-F B_{n n}\right)-{ }_{3}^{4} A B_{n l} B_{n n} \\
& +\frac{2}{9} P B_{n n} B_{n n}-\frac{2}{3}(A B-2 C) B_{n n}^{2} \text {, } \\
& 3 S=-4 A_{n} E\left(\frac{2}{3} B B_{n n}+B_{n l}\right)+8 A_{m l}\left(E B_{m n}-F B_{n n}\right)+\frac{2}{3} B_{n n} B_{n l} \\
& -\frac{4}{3} A B_{n n} B_{n n}+{ }_{8}^{8} B B_{n n}^{2} \text {, } \\
& T=-\frac{1}{4} A_{l l}\left(E B_{n n 2}-F B_{n n}\right)+{ }_{3}^{2} B_{n m} B_{n n}-\frac{2}{i} A B_{n n 2}^{2} \text {. }
\end{aligned}
$$

Si ha dunque il teorema :

Se $R=0$, senza che sia nullo anche $A_{q g}$, la forma $u$ si puo rappresentare come funzione razionale di terzo grado in $l, m$.

Ne risulta che in questo caso l'equazione di sesto grado $u=0$ puó essere risoluta nel modo seguento. Mediante la sostituzione di secondo grado:

$$
z=m,
$$


l'equazione si riduce a quest'altra di terzo grado:

$$
P+3 Q z+3 S z^{2}+T z^{3}=0 \text {. }
$$

Se $z=\zeta$ è una radice di questa equazione, si avrà inoltre :

$$
\curvearrowright=l \sqrt{-\left(A_{n l} \zeta^{2}-2 A_{l m} \zeta+A_{n n}\right)}
$$

Siccome ora i rapporti fra $l, m, \downarrow$ sono affatto determinati, cosi si possono scrivere le equazioni:

$$
l=\mu \cdot \alpha, \quad \cdots=\mu \cdot \beta, \quad \$=\mu \cdot \gamma,
$$

ove $\mu$ è un fattore indeterminato ed $\alpha, \beta, \gamma$ esprimono numeri dati. Queste tre equazioni sono lineari rispetto ad $x_{1}^{2}, x_{1} x_{2}, x_{2}^{2}$; e risolvendole si trovano le radici dell' equazione data.

Il caso presente è quello nel quale le radici dell' equazione $u=0$ formano un' involuzione (1). In fatti, se $A_{\vartheta}$ y non si annulla, $l$ ed $m$ possono essere trasformati simultaneamente, mediante una sostituzione lineare, in somme di quadrati, ed $u=0$ si trasforma in un'equazione che del pari contiene soltanto i quadrati delle variabili : il che è il segno distintivo dell'involuzione. Viceversa, se vi è involuzione, si ha sempre $R=0$. In fatti, supposto che $u$ contenga solamente i quadrati delle variabili, è facile vedere che anche $k$ conterrà i soli quadrati, e la medesima cosa dovrà verificarsi per $l, m, n$. Quindi fra $l, m, n$ avlà luogo una relazione lineare e per conseguenza il determinante $R$ di queste funzioni dovrà ridursi a zero.

\section{$\$ 12$.}

secondo caso ecceziomale : $R=0, A_{j} y=0$.

Le due rappresentazioni tipiche che abbiamo date precedentemente divengono impossibili quando, oltre $R$, si annulli anche $A \vartheta \vartheta$. Per mezzo dell'equazione :

$$
A_{\vartheta \vartheta}=A_{l b} A_{m m}-A_{l m}^{2}=0,
$$

(1) Yedi Salaion, Higher Algebra, p. $210\left(2 .^{\mathrm{a}}\right.$ ediz.) 
la $R^{2}=0$ si muta in:

$$
A_{l l} A_{m i l}-A_{l m} A_{l n}=0
$$

ossia :

$$
A_{m l} A_{n n n}-A_{m m} A_{l n}=0 \text {. }
$$

Siccome $A_{l n}=A_{n n a}$, così si possono determinare due numeri $\lambda, \mu$ in modo che :

$$
A_{l l}=\lambda^{4}, A_{l n}=\lambda^{3} \mu, A_{l n}=A_{m n}=\lambda^{2} \mu^{2}, A_{m n}=\lambda \mu^{3},
$$

e le equazioni :

$$
A_{n m}=B A_{l i l}+2 C A_{l l}, \quad A_{n n}=B A_{m m}+2 C A_{m l}
$$

diverranno allora :

$$
\mu^{3}=B \lambda^{2} \mu+2 C \lambda^{3}, \quad A_{m}=\left(B \lambda^{2} \mu+2 C \lambda^{3}\right) \mu=\mu^{4} .
$$

Gli invarianti $A_{l l}, A_{l m}, \ldots, A_{2 n}$ sono dunque i coefficienti di un biquadrato perfetto $(\lambda x+\mu y)^{4}$; e la quantità $\frac{\mu}{\lambda}$ sodisfà all'equazione cubica :

$$
\left(\frac{\mu}{\lambda}\right)^{3}-B \frac{\mu}{\lambda}-2 C=0
$$

Poi le equazioni che esprimono $A_{l l}, A_{l m}$ per mezzo di $A, B, C$, divengono:

$$
\lambda^{4}=2\left(C+{ }_{3}^{1} A B\right), \quad \lambda^{3} u=4\left(A C+\frac{1}{9} B^{3}\right),
$$

donde si ricava:

$$
\frac{\mu}{\lambda}=2 \frac{A C+\frac{1}{9} B^{2}}{C+\frac{1}{3} A B^{2}}
$$

e introducendo questo valore nell' equazione cubica superiore, si ottiene la relazione seguente fia $A, B, C$.

$$
\begin{aligned}
0 & =\left(C+\frac{1}{3} A_{1 l}\right)^{3} C+B\left(A C+\frac{1}{9} B^{2}\right)\left(C+\frac{1}{3} A B\right)^{2}-4\left(A C+\frac{1}{9} B^{2}\right)^{2} \\
& =\left(C^{2}-{ }_{27}^{1} B^{3}\right)\left\{C^{2}-4 A^{3} C+2 A B C-\frac{1}{3} A^{2} B^{2}+\frac{4}{27} B^{3}\right\} .
\end{aligned}
$$

Qui si vede che sono a distinguersi due casi secondo che si annulli il primo o il secondo fattore. Alla medesima conseguenza si giunge anche per 
altra via, che manifesta a un tempo il significato dell' aunullarsi di questi fattori. L'equazione $A_{\vartheta}=0$, in virtủ della quale \& è un quadrato perfetto, indica evidentemente che le forme quadratiche $l, m$ posseggono un fattor comune. Se questo fattore di primo grado, onde $l=a . b, m=a . c$ (dove $a, b, c$ sono espressioni lineari, e $b$ è diverso da $c$ ), l'equazione $R=0$ dice che fra $l, m, n$ sussiste una relazione lineare, e che per conseguenza anche $n$ contiene il fattore $a$; sia $n=a . d$. Invece, se quel fattore comune di $l$ ed $m$ è di secondo grado, sarà $m$ proporzionale ad $l$, ossia $m=\varepsilon . l$, e allora l'equazione $R=0$ è sodisfatta da sè. Siccome poi si ha:

$$
n=k_{11} m_{22}-2 k_{12} m_{12}+k_{22} n_{11}=\varepsilon\left(k_{11} l_{22}-2 k_{12} l_{12}+k_{z 2} l_{11}\right)=\varepsilon . m=\varepsilon^{2} . l,
$$

così anche $n$ è proporzionale ad $l$.

Cominciamo dal considerare il primo caso. Posto per brevitá:

$$
k_{1} a_{2}-k_{2} a_{1}=k^{\prime}
$$

si ha:

$$
\begin{aligned}
& k_{11} l_{22}-2 k_{12} l_{12}+k_{22} l_{11}=k_{1}^{\prime} b_{2}-k_{2}^{\prime} b_{1}=m=a c, \\
& k_{11} m_{22}-2 k_{12} m_{12}+k_{22} m_{11}=k_{1}^{\prime} c_{2}-k_{2}^{\prime} c_{1}=n=a d .
\end{aligned}
$$

L'equazione identica :

dà pertanto:

$$
0=\left|\begin{array}{lll}
k^{\prime} & b & c \\
k_{1}^{\prime} & b_{1} & c_{1} \\
k_{2}^{\prime} & b_{2} & c_{2}
\end{array}\right|
$$

$$
0=k^{\prime}\left(b_{1} c_{2}-c_{1} b_{2}\right)+a\left(c^{2}-b d\right) .
$$

Di qui segue che $k^{\prime}$ è divisibile per $a$, epperỏ $=a k^{\prime \prime}$. Ma introducendo questo valore nelle equazioni precedenti si ottiene:

$$
\begin{aligned}
& 3 a c=k^{\prime \prime}\left(a_{1} b_{2}-b_{1} a_{2}\right)+2 a\left(k_{1}^{\prime \prime}{ }_{1} b_{2}-k_{2}^{\prime \prime}{ }_{2} b_{1}\right), \\
& 3 a d=k^{\prime \prime}\left(a_{1} c_{2}-c_{1} a_{2}\right)+2 a\left(k_{1}^{\prime \prime}{ }_{1} c_{2}-k_{2}^{\prime \prime} c_{1}\right),
\end{aligned}
$$

e siccome in ogni caso o le $b$ o le $c$ sono diverse dalle $a$, cosi risulta di qui che $k^{\prime \prime}$ è ancora divisibile per $a$, cioè $k^{\prime}=a^{2} e$, dove ora $e$ è una funzione 
lineare. Posto per $k^{\prime}$ questo valore, le equazioni superiori riescono divisibili per $a$, e servono a definire $c$ e $d$ mediante $a, b, e$.

Ora dall' equazione :

$$
k_{1} a_{2}-k_{2} a_{1}=u^{2} e
$$

segue, se $\check{\zeta}$ è una funziono lineare qualunque differente da $a$ :

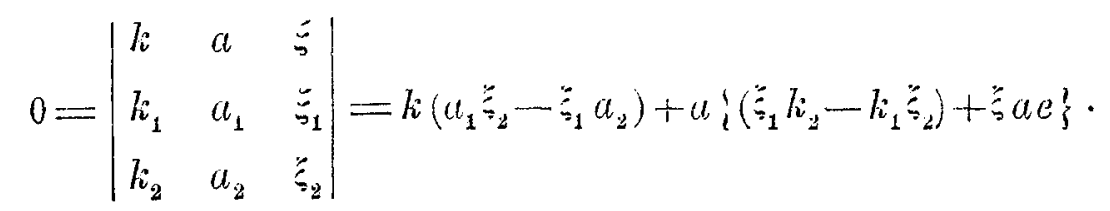

Dunque $k$ è divisibile per $a$, cioè $k=a . s$; onde la nostra equazione diviene :

$$
0=3 s\left(a_{1} \zeta_{2}-\zeta_{1} a_{2}\right)+a\left\{3\left(\zeta_{1} s_{2}-s_{1} \zeta_{2}\right)+4 \zeta c\right\} \text {. }
$$

Quindi $s$ è di nuovo divisibile per $a$; e posto $k=a^{2} . r$, l' equazione precedente acquista il fattore $a$. Dunque $k$ contiene due fattori eguali al fattore comune di $l$ ed $m$.

Passando ora alla forma $u$, abbiamo le equazioni seguenti, nelle quali si è posto $u \iota^{\prime}=u_{1} a_{2}-a_{1} u_{2}$ :

$$
\begin{aligned}
& u_{11} l_{22}-2 u_{12} l_{12}+u_{22} l_{11}=u_{1}^{\prime} b_{2}-u_{2}^{\prime} b_{1}=\frac{2}{3} \Delta+\frac{9}{3} A k, \\
& u_{11} m_{22}-2 u_{12} m_{12}+u_{22} m_{11}=u_{1}^{\prime} c_{2}-u_{2}^{\prime} c_{1}=\frac{1}{2} l^{2}+\frac{9}{3} B l_{i}+\frac{2}{3} A \Delta .
\end{aligned}
$$

Ma la funzione $\Delta$ è, insieme con $k$, divisibilo per $a^{2}$; epperò questo fattore è comune ai due termini del secondo membro dell' equazione. Si dimostrerà adunque, nello stesso modo come si è fatto sopra per $k$, che $u^{\prime} \dot{0}$ divisibile per $a^{3}$, ciò̀ $u^{\prime}=a^{3} \cdot f$, ed allora dall' equazione:

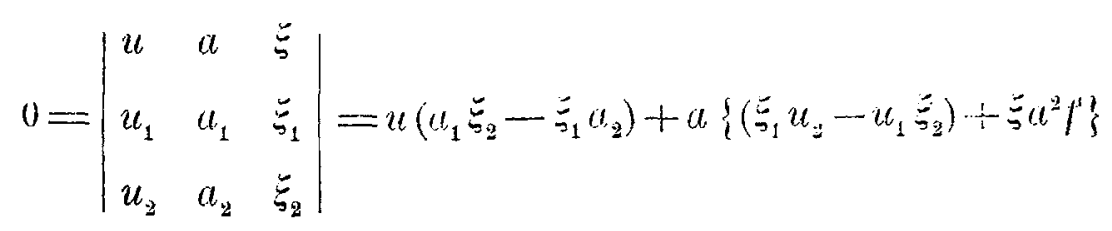

segue che anche $u$ contiene il fattore $a^{3}$. Con ciò è dimostrato il teorema:

Se $l$ ed $m$ hanno un fattore comune, però senza essereproporzionali fra loro, questo fattore è contenuto al quadrato in $k$ ed al cubo in $u$. 
Dalla prima proprietà s'inferisce che in questo caso il discriminante di $k$ è nullo, ciò̀ :

$$
C^{2}-\frac{1}{27} B^{3}=0
$$

dove il primo membro è il primo de'due fattori dell'equazione sopra incontrata.

Il teorema ora enunciato si può anche invertire. Se $a$ è una funzicne lineare e $w$ una funzione cubica, entrambe qualisivogliano, e se $u=a^{3} w$, sarà $k$ sempre divisibile per $a^{2}$, ed $l, m$ divisibili per $a$. In fatti, assunti $a$ e $\xi$ come variabili, e formato $k$ rispetto a queste variabili, si ha per $k$, astrazion fatta da un fattore costante, l'espressione:

$$
\frac{\partial^{4} \cdot a^{3} w}{\partial a^{4}} \cdot \frac{\partial^{4} \cdot a^{3} w}{\partial \zeta^{4}}-4 \frac{\partial^{4} \cdot a^{3} w}{\partial a^{3} \cdot \partial^{3}} \cdot \frac{\partial^{4} \cdot a^{3} w}{\partial a \cdot \partial \partial^{3}}+3\left(\frac{\partial^{4} \cdot a^{3} w}{\partial a^{2} \cdot \partial z^{2}}\right)^{2}
$$

nella quale il primo termine $\dot{e}$ nullo, e ciascuno degli altri $\dot{e}$ divisibile per $a^{2}$. Quindi $k=a^{2} r$. Se ora formiamo anche $l$ rispetto alle variabili $a, \xi$, otteniamo l'espressione:

$$
\begin{aligned}
\frac{\partial^{4} \cdot a^{2} r}{\partial a^{4}} \cdot \frac{\partial^{4} a^{3} w}{\partial \xi^{4}} & -4 \frac{\partial^{4} \cdot a^{2} r}{\partial a^{3} \cdot \partial \xi} \cdot \frac{\partial^{4} \cdot a^{3} w}{\partial a \cdot \partial \xi^{3}}+6 \frac{\partial^{4} \cdot a^{2} r}{\partial a^{2} \cdot \partial \xi^{2}} \cdot \frac{\partial^{4} \cdot a^{3} w}{\partial a^{2} \cdot \partial \xi^{2}} \\
& -4 \frac{\partial^{4} \cdot a^{2} r}{\partial a \cdot \partial \xi} \cdot \frac{\partial^{4} \cdot a^{3} w}{\partial a^{3} \cdot \partial \xi}+\frac{\partial^{4} \cdot a^{2} r}{\partial \xi^{4}} \cdot \frac{\partial^{4} \cdot a^{3} w}{\partial a^{4}},
\end{aligned}
$$

che è divisibile per $a$. La stessa cosa ha luogo per l'espressione di $m$ :

$$
\frac{\partial^{2} \cdot a^{2} r}{\partial a^{2}} \cdot \frac{\partial^{2} l}{\partial \xi^{2}}-2 \frac{\partial^{2} \cdot a^{2} r}{\partial a \cdot \partial \xi} \cdot \frac{\partial^{2} l}{\partial \xi \cdot \partial a}+\frac{\partial^{2} \cdot a^{2} r}{\partial \xi^{2}} \cdot \frac{\partial^{2} l}{\partial a^{2}}
$$

come anche per l'espressione analoga di $n$. Dunque possiamo enunciare il teorema :

Se $u$ contiene un fattore cubico, questo entra al quadrato in $k$ e linearmente in $l, m, n$, e sono nulli gl' invarianti $R$ e $C^{2}-\frac{1}{2} B^{3}$.

Passiamo ora all'altro caso nel quale $m$ ed $l$ non differiscono che per un fattore costante $z$. Per le cose precedenti si ha in questo caso:

$$
\begin{gathered}
m=\varepsilon l, n=\varepsilon^{2} l, \\
A_{m l}=\varepsilon A_{l l}, \quad A_{2 n m}=A_{n t}=\varepsilon^{2} A_{n}, \quad A_{m n}=\varepsilon^{3} A_{l l}, \quad A_{n n}=\varepsilon^{4} A_{l l}, \\
\varepsilon^{3}-D z-2 C=0, \quad c\left(C+\frac{1}{3} A B\right)-2\left(A C+\frac{1}{9} B^{2}\right)=0 .
\end{gathered}
$$


Le equazioni per $k, \Delta$ divengono:

$$
\begin{aligned}
& k_{11} l_{22}-2 k_{12} l_{12}+k_{22} l_{11}=\varepsilon l, \\
& \Delta_{11} l_{22}-2 \Delta_{12} l_{12}+\Delta_{22} l_{11}=\left(\varepsilon^{2}-\frac{2}{3} B\right) l,
\end{aligned}
$$

mentre dall' equazioni per $u$ si ottiene:

$$
\begin{aligned}
u_{11} l_{22}-2 u_{12} l_{12}+u_{22} l_{11} & =\frac{2}{3} A k+\frac{2}{3} \Delta \\
& =\frac{1}{\varepsilon}\left(\frac{1}{2} l^{2}+\frac{2}{9} B k+\frac{2}{3} A \Delta\right) \\
& =\frac{1}{\varepsilon^{2}}\left(\varepsilon l^{2}-\frac{2}{3} C k+\frac{2}{9} B \Delta\right) .
\end{aligned}
$$

Da queste ultime segue 0 che $k$ e $\Delta$ non differiscono da $l^{2}$ che per un fattore, o che dev'essere nullo il determinante:

$$
\left|\begin{array}{ccc}
0 & A & 1 \\
\frac{1}{2} & \frac{2}{9} B & \frac{2}{3} A \\
\varepsilon & -\frac{2}{3} C & \frac{2}{4} B
\end{array}\right|=\frac{2}{3} \varepsilon\left(A^{3}-\frac{1}{3} B\right)-\frac{1}{3}\left(C+\frac{1}{3} A B\right) .
$$

Nell' ultimo caso, sostituendo il valore:

$$
\varepsilon=2 \frac{A C+\frac{1}{9} B^{2}}{C+1 A B}
$$

si ottiene la condizione:

$$
C^{2}-4 A^{3} C+2 A C B-\frac{1}{3} A^{2} B^{2}+\frac{4}{27} B^{3}=0,
$$

dove il primo membro è il secondo dei fattori dell'equazione sopra ricordata. In questo caso che noi tratteremo pel primo, si può rappresentare simultaneamente $B$ e $C$ come funzioni intere di $A$ ed $\varepsilon$, in modo che la predetta condizione sia sodisfatta identicamente. In fatti dalle equazioni :

$$
\varepsilon^{3}-B \varepsilon-2 C=0,2 \varepsilon\left(A^{2}-\frac{1}{3} B\right)-\left(C+\frac{1}{3} A B\right),
$$

segue :

$$
B=3 \varepsilon(2 A-\varepsilon), C=2 \varepsilon^{3}-3 A \varepsilon^{2} .
$$

Siccome inoltre :

$$
A_{l l}=2 C+\frac{2}{3} A B=4 \varepsilon\left(\varepsilon-A^{2}\right)
$$


così tutti gli invarianti sono espressi come funzioni intere di $A, \varepsilon$.

Le equazioni sopra riferite per $u$ danno:

$$
\frac{3}{4} l^{2}=\frac{\varepsilon-A}{\varepsilon}(\Delta+\varepsilon k) .
$$

In fatti, per la teoria delle forme biquadratiche: le tre quantità $\lambda$ che rendono $\Delta+\lambda k$ un quadrato perfetto sono date dall'equazione cubica:

$$
\lambda^{3}-B \lambda-2 C=0,
$$

che, per le cose precedenti, ha la radice $\varepsilon$. Si vede poi dall'equazione precedente che per $\lambda=\varepsilon$ la radice del quadrato perfetto differisce da $l$ soltanto di un fattore costante.

Imaginiamo ora che $l$ sia da prima il prodotto di due fattori disuguali. Assumendo questi come variabili $x, y$, onde $l=2 x y$, l'equazione:

diviene :

$$
k_{11} l_{22}-2 k_{12} l_{12}+k_{22} l_{11}=\varepsilon l
$$

epperò :

$$
-k_{12}=\varepsilon \cdot x y
$$

$$
k=p x^{4}-3 \varepsilon x^{2} y^{2}+q y^{4}
$$

dove $p$ e $q$ sono due coefficienti ancora incogniti, fra i quali pero ha luogo l'equazione :

$$
p q=6 \mathrm{~A} \varepsilon-\frac{15}{1} \varepsilon^{2}
$$

risultante dalle definizioni di $B$ e $C$. Inoltre l'equazione:

diviene :

$$
u_{11} l_{22}-2 u_{12} l_{12}+u_{22} l_{11}=\frac{2}{3}(A k+\Delta)
$$

$$
-3 u_{12}=(A-\varepsilon)\left\{p x^{4}+q y^{4}+9 \varepsilon x^{2} y^{2}\right\}
$$

onde :

$$
u=p^{\prime} x^{6}+q^{\prime} y^{6}-2(A-\varepsilon)\left\{\left(p x^{4}+q y^{4}\right) x y+5 \varepsilon x^{3} y^{3}\right\}
$$

Si osservi che qui mancano i termini $x^{4} y^{2}$ ed $x^{2} y^{4}(c=0, e=0)$. Se ora si scrivono i coefficienti di $k$ e si pongono, come dev'essere, il secondo ed il penultimo eguali a zero, si scorge che il discriminante di $k$ e diverso 
da zero solamente quando $p^{\prime}$ e $q^{\prime}$ sono nulli. Ma se è nullo il discriminante di $k$, si annullano entrambi i fattori dell'equazione di condizione superiormente decomposta: caso che sarà considerato più avanti.

Di qui si vede che se deve annullarsi soltanto il secondo di quei fattori, $u$ diviene il prodotto $x y$ moltiplicato per una funzione pari di quarto grado, ed è facile mostrare che una forma $u$ così fatta conduce sempre ad un covariante $k$ che contiene le sole potenze pari, e che $l$ è proporzionale ad $m$. Per valutare la scelta speciale delle variabili, si noti che $x^{2} y^{2}$ è uno dei tre quadrati nei quali può essere trasformata una somma della funzione di quarto grado e del suo determinante. Abbiamo dunque il teorema:

Sia $\phi$ una funzione binaria di quarto grado ed $l^{2}$ uno dei quadrati nei quali puo essere trasformata l'espressione $\Delta_{\varphi}+\lambda \phi$. Allora $\phi . l$ è l'unica funzione di sesto grado per la quale $A_{\vartheta}$ ed $R$ si annullano in modo che $l$ sia proporzionale ad $m$ e chel'espressione:

$$
C^{2}-4 A^{3} C+2 A C B-\frac{1}{3} A^{2} B^{2}+\frac{1}{27} B^{3}
$$

sia nulla, senza che vada a zero anche il discriminante di $k$.

Per completare la dimostrazione del teorema, si deve ancora mostrare che, se $l$ è un quadrato, sotto le predette condizioni il discriminante di $k$ ¿̀ zero. In fatti, per ciò che si è detto sopra, allora è zero $A_{l l}$, epperò anche $\varepsilon \quad 0 \quad \varepsilon-A$. Quindi o ̀̀ $B=0, G=0$, oppure $B=3 \varepsilon^{2}, G=-\varepsilon^{2}$, ciò̀ $B^{3}=27 C^{2}$, ciỏ che si doveva dimostrare.

Casi ulteriori che risultano dall'annullarsi identicamente di $m \quad 0$ di $l$ saranno trattati in seguito.

Del resto la equazione di condizione sopra considerata, insieme con $R=0$, è equivalente ad $E=0, F=0$; perchè essa è il risultato dell' eliminazione di $D$ da queste ultime equazioni.

Passiamo ora all'altro caso che si ricava dalla proporzionalità di $m$ ed $l$. In questo caso $k$ e $\Delta$ erano proporzionali ad $l^{2}$. Possiamo in cid che precede cominciare dal supporre che $l$ non sia un quadrato, $l=2 x y$, e troviamo allora $k, \Delta$ proporzionali ad $x^{2} y^{2}$. Quindi anche $u_{12}$ ¿ proporzionale ad $l^{2}$, cioè $u$ assume la forma :

$$
u=p x^{6}+20 q x^{3} y^{3}+r y^{6}
$$


In fatti, presupponendo questa forma per $u$, si trova il covariante $k$ proporzionale ad $x^{2} y^{2}$ ed $l$ proporzionale ad $x y$. Si ha cosi il teorema:

Se $m$ è proporzionale ad $l$, e se $k$ è proporzionale al quadrato di $l$, la forma $u$ è riducibile, per mezzo di una sostituzione lineare, ad una funzione quadratica di cubi.

Se in questo caso $l$ è un quadrato, $l=x^{2}$, avremo $k$ e $\Delta$ proporzionali ad $x^{4}$, quindi $m=0$, il che sarà più avanti sogrgetto di speciale ricerca.

Passiamo ora ai casi ne' quali si annullano entrambi $i$ fattori :

$$
B^{3}-27 C^{2}, C^{2}-4 A^{3} C+2 A C B-\frac{1}{3} A^{2} B^{2}+\frac{1}{27} B^{3} .
$$

Allora introducendo nella prima condizione i valori:

$$
B=3 \varepsilon(2 A-\varepsilon), C=\varepsilon^{2}(2 \varepsilon-3 A),
$$

che sodisfanno alla seconda, si trova :

$$
\begin{aligned}
\left(\frac{1}{3} B\right)^{3}-C^{2} & =\varepsilon^{3}\left\{8 A^{3}-21 A^{2} \varepsilon+18 A \varepsilon^{2}-5 \varepsilon^{3}\right\} \\
& =\varepsilon^{3}(A-\varepsilon)^{2}(8 A-5 \varepsilon) .
\end{aligned}
$$

Fatta astrazione dal caso $\varepsilon=0$ che conduce ad $m=0$, e da $A=\varepsilon$ che, a cagione dell' equazione :

$$
l^{2}=\frac{\varepsilon-A}{\varepsilon}(\Delta+\varepsilon k),
$$

conduce ad $l=0$, non rimane che il caso:

$$
A={ }_{8}^{5} \varepsilon, \quad B=\frac{3}{4} \varepsilon^{2}, \quad C=\frac{1}{8} \varepsilon^{3} .
$$

Qui possiamo supporre che $A_{l l}=4 \varepsilon(\varepsilon-A)^{2}=\frac{9}{16} \varepsilon^{3}$ non sia zero, epperò che $l$ abbia due fattori differenti. Posto adunque $l=2 x y$, segue come sopra:

$$
k=p x^{4}+q y^{4}-3 \varepsilon x^{2} y^{2},
$$

colla condizione $p . q=0$. Sia p. e. $q=0$; allora:

$$
k=p x^{4}-3 \varepsilon y^{2} x^{2}, \quad \Delta=-p \varepsilon x^{4}-\frac{3}{2} \varepsilon^{2} x^{2} y^{2},
$$

quindi :

$$
u_{11} l_{22}-2 u_{12} l_{12}+u_{22} l_{11}=-\frac{1}{15} \frac{\partial^{2} u}{\partial x \partial y}=-{ }_{8}^{3} \varepsilon p x^{4}-\frac{\eta 7}{3} \varepsilon^{2} x^{2} y^{2} .
$$

Per conseguenza ‘ ha la forma:

$$
u=a x^{6}+6 b x^{5} y+20 d x^{3} y^{3}+9 y^{6} \text {. }
$$


Ricavando di qui $k$, si trova :

$$
k=-24 b d x^{4}+6\left(a g+8 d^{2}\right) x^{2} y^{2}+12 b g x y^{3} .
$$

Affinchè questa espressione coincida colla forma precedente di $k$, deve essere zero $b g$. Ma siccome $A=a g-10 d^{2}, B=\left(a g+8 d^{2}\right)^{2}$, cosi la condizione $\frac{4}{3} B-\left(\frac{8}{3} A\right)^{2}$ per $g=0$ non è verificata che per $d=0$, donde $k=0$ : caso del quale sarà trattato più sotto. Facendo invece $b=0$, si ha un caso speciale incluso in quello dianzi considerato, nel quale $u$ è rappresentabile come funzione quadratica di cubi lineari.

\section{$\S 13$.}

Aicerea del casi : $m=0, l=0, k=0$.

Sinora abbiamo esauriti tutti i casi particolari ne' quali $m$ non si annulla. Se ciò ha luogo, si annullano a dirittura anche gli invarianti :

$$
R, A_{m l}, A_{m n}, A_{m n}, A_{n n},
$$

e si annullano inoltre le forme $n$ e

$$
p=B m+2 C l \text {. }
$$

Se $l$ non dev'essere zero, sarà $G=0$. Dalla:

$$
0=A_{m b}=\frac{4}{5} B^{2}+4 A C
$$

allora segue $B=0$, e quindi anche:

$$
A_{l l}=2 C+\frac{2}{3} A B=0 \text {. }
$$

Dunque $l$ è un quadrato, $l=\rho x^{2}$. Dall' equazione :

$$
k_{11} l_{22}-2 k_{12} l_{12}+k_{22} l_{11}=0,
$$

abbiamo per $k$ la determinazione $k_{22}=0$, onde:

e simultaneamente:

$$
k=p x^{4}+4 q x^{3} y
$$

$$
\Delta=-2 q^{*} x^{2}=\frac{3}{24}\left\{u_{11} n_{2: 2}-2 u_{12} m_{12}+u_{22} i u_{11}-\frac{1}{2} l^{2}\right\}=-\frac{3 \rho^{2}}{4 A} x^{4}
$$

epperò :

$$
q^{2}=\frac{3}{8} \frac{p^{2}}{I},
$$


se non è $A=0$, donde seguirebbe $\frac{1}{2} l^{2}=-\frac{9}{3} A \Delta=0$.

Inoltre dalla:

$$
u_{11} l_{22}-2 u_{12} l_{12}-u_{22} l_{11}=\frac{2}{3}(\Delta+A k),
$$

segue l'equazione:

$$
\frac{p}{20} \frac{\partial^{2} u}{\partial x^{2}}=\left(A p-2 q^{2}\right) x^{4}+4 A q x^{3} y ;
$$

quindi, se non è zero $\rho$ cioè $l$, si avià per $u$ la forma :

$$
u=a x^{6}+6 b x^{5} y+6 f x y^{5}+g y^{6} \text {. }
$$

Ricavando di qui $k$, si ottiene :

$$
k=12 a f x^{3} y+6 a g x^{2} y^{2}+12 b g x y^{3} .
$$

Per tal modn si vede che nelle formole antecedenti dev'essere $p=0$, ed inoltre $a g=0, b g=0$. O sono nulli $a$ e $b$, epperò anche $k$; o è zero $g$, ed allora :

Quindi :

$$
u=a x^{6}+6 b x^{5}+6 f x y^{5}, \quad k=12 a f x^{3} y .
$$

$$
l=-4 u_{1222} k_{112}=-12 a f^{2} y^{2},
$$

ciò che contradice al valore assunto per $l$. Dunque è in ogni casc $l=0$, e si ha il teorema :

Se il covariante $m$ si annulla identicamente, è identicamente zero anche il covariante $l$.

Rimane dunque ad esaminarsi solamente in quali casi $l$ è zero; cominciando a supporre che $k$ non si annulli. Siccome in questo caso:

$$
\Delta+A k=\frac{3}{2}\left(u_{11} l_{22}-2 u_{12} l_{12}+u_{22} l_{11}\right)=0 \text {, }
$$

cosi $k$ è un quadrato perfetto. Per conseguenza si puó porre $k=6 x^{2} y^{2}$ oppure $k=x^{4}$. Nel primo caso l'equazione che definisce $l$ per mezzo di $k$ ed $u$ diviene $u_{1122}=0$, ossia :

$$
c x^{2}+2 d x y+e y^{2}=0 .
$$

Dunque $c=d=e=0$, eppero :

$$
u=a x^{6}+6 b x^{5} y+6 f x y^{5}+g y^{6}
$$

Ricavando $k$, si trova:

$$
k=12 a f x^{3} y+6 a g x^{2} y^{2}+12 b g x y^{3} \text {. }
$$


Perciò dev' essere $a f=0, b g=0 ;$ quindi, se $k$ non deve andare a zero, $b=0, f=0$. Dunque $u$ è una somma di seste potenze.

In secondo luogo, se $k=x^{4}$, l'equazione che definisce $l$ per mezzo di $u$ e $k$ dà $u_{2222}=0$, dunque:

donde segue :

$$
u=a x^{6}+6 b x^{5} y+15 c x^{4} y^{2}+20 d x^{3} y^{3}
$$

$$
k=6\left(3 c^{2}-4 b d\right) x^{4}+24 c d x^{3} y+8 d^{2} x^{2} y^{2} .
$$

Perchè questa forma di $k$ coincida colla prima, dev' essere $d=0$, onde:

$$
u=a x^{6}+6 b x^{5} y+15 c x^{4} y^{2} \text {. }
$$

E si ha cosi il teorema:

Se $l$ è zero, o $u$ è una somma di due seste potenze, ovvero $u$ contiene una radice quadrupla; ed in entrambi i casi si annulla sempre anche $l$.

Finalmente restano soltanto i casi ne' quali $k$ è identicamente nullo. Ciò si verifica in primo luogo, evidentemente, se $u$ è una sesta potenza. Se $u$ non ha questa forma, si possono assumere come variabili due diversi fattori di $k$, onde si avrà $a=0, g=0$; ed affinchè allora $k$ si annulli, devono essere sodisfatte le equazioni:

$$
\begin{gathered}
4 b d-3 c^{2}=0 \quad, \quad 3 b e-2 c d=0, \\
4 d f-3 e^{2}=0 \quad, \quad 3 c f-2 d e=0 \\
9 c e-8 d^{2}=0 .
\end{gathered}
$$

Se in primo luogo è $d=0$, anche $c$ ed $e$ devono essere zero; quindi $u$ ha la forma:

$$
u=6\left(x^{5} y+y^{5} x\right)
$$

Ma se $d$ è diverso da zero, nessuno dei coefficienti $c, e, b, f$ può essere nullo, ed esprimendo tutto mediante $b, c$, si trova:

$$
\begin{gathered}
u=\frac{3 x y}{2 b^{3}}\left\{4 b^{4} x^{4}+10 c b^{3} x^{3} y+10 c^{2} b^{2} x^{2} y^{2}+5 c^{3} b x y^{3}+c^{4} y^{4}\right\} \\
=\frac{3 x(c y+b x)}{2 b^{3} c}\left\{(c y+b x)^{4}-b^{4} x^{4}\right\},
\end{gathered}
$$

espressione che mediante una sostituzione lineare si reca del pari alla forma $6 x y\left(x^{4}+y^{4}\right)$. 
La forma $6 x y\left(x^{4}+y^{4}\right)$ è il covariante di sesto ordine di una forma biquadratica :

$$
x^{4}+y^{4}+6 \lambda x^{2} y^{2} .
$$

Si può adunque enunciare il teorema :

Se per una forma binaria $u$ di sesto grado il covariante $k$ è identicamente nullo, u sarà una sesta potenza, ovvero sarà il covariante di sesto grado di una forma biquadratica; e viceversa $k$ si annulla identicamente in ambedue questi casi.

Per risolvere l'equazione $u=0$ nel caso che $k$ sia nullo, basta fare l'osservazione che i fattori di $6 x y\left(x^{4}+y^{4}\right)$, cioè :

$$
2 x y, \quad x^{2}+y^{2} i, \quad x^{2}-y^{2} i
$$

danno sempre zero, se si formano gli invarianti simultanei di due qualunque fra essi. Dunque, se i fattori di $u$ si indicano con:

$$
x^{2}+2 \alpha x y+\beta y^{2}, \quad x^{2}+2 \alpha^{\prime} x y+\beta^{\prime} x^{2}, \quad x^{2}+2 \alpha^{\prime \prime} x y+\beta^{\prime \prime} y^{2}
$$

questi possederanno la medesima proprietà, onde:

$$
\beta+\beta^{\prime}=2 \alpha \alpha^{\prime}, \quad \beta^{\prime}+\beta^{\prime \prime}=2 \alpha^{\prime} \alpha^{\prime \prime}, \quad \beta^{\prime \prime}+\beta=2 \alpha^{\prime \prime} \alpha .
$$

I coefficienti del prodotto di quei tre fattori diventano percio i seguenti:

1, $2\left(\alpha+\alpha^{\prime}+\alpha^{\prime \prime}\right), \quad \beta+\beta^{\prime}+\beta^{\prime \prime}+4\left(\alpha \alpha^{\prime}+\alpha^{\prime} \alpha^{\prime \prime}+\alpha^{\prime \prime} \alpha\right)=5\left(\beta+\beta^{\prime}+\beta^{\prime \prime}\right)$,

$2\left(\alpha^{\prime \prime} \beta^{\prime}+\cdot \cdot\right)+8 \alpha \alpha^{\prime} \alpha^{\prime \prime}, \quad 4\left(\alpha \alpha^{\prime} \beta^{\prime \prime}+\alpha^{\prime} \alpha^{\prime \prime} \beta+\alpha^{\prime \prime} \alpha \beta^{\prime}\right)+\beta^{\prime} \beta^{\prime \prime}+\beta^{\prime \prime} \beta+\beta \beta^{\prime}=$

$$
=5\left(\beta \beta^{\prime}+\beta^{\prime} \beta^{\prime \prime}+\beta^{\prime \prime} \beta\right), \quad 2\left(\alpha \beta^{\prime} \beta^{\prime \prime}+\alpha^{\prime} \beta^{\prime \prime} \beta+\alpha^{\prime \prime} \beta \beta^{\prime}\right), \quad \beta \beta^{\prime} \beta^{\prime \prime} \text {. }
$$

Dunque, se è data la forma:

$$
u=a x^{6}+6 b x^{5} y+15 x^{4} y^{2}+20 d x^{3} y^{3}+15 e x^{2} y^{4}+6 f x y^{5}+g y^{6},
$$

si ricavano lo $\beta$ dall' equazione cubica :

$$
a \beta^{3}-3 c \beta^{2}+3 e \beta-q=0,
$$

e quindi le $\alpha$ mediante equazioni lineari.

Giessen, feblurajo $186 \%$. 